\title{
DIHEDRAL COVERINGS OF TRIGONAL CURVES
}

\author{
Alex Degtyarev
}

\begin{abstract}
We classify and study trigonal curves in Hirzebruch surfaces admitting dihedral Galois coverings. As a consequence, we obtain certain restrictions on the fundamental group of a plane curve $D$ with a singular point of multiplicity (deg $D-3$ ).
\end{abstract}

\section{INTRODUCTION}

1.1. Motivation. This paper begins a systematic study of the fundamental group of a trigonal curve in a geometrically ruled rational surface. The main tools used are the braid monodromy, Zariski-van Kampen theorem, and arithmetic properties of the modular group $\Gamma:=P S L(2, \mathbb{Z})$.

Originally, my interest in trigonal curves was motivated by my attempts to compute the fundamental groups of plane curves, a problem that was first posed by O. Zariski [20], [21] in 1930 and that has since been a subject of intensive research by a number of mathematicians. Given such a curve $D \subset \mathbb{P}^{2}$, one can blow up a singular point $P$ and, by a sequence of elementary transformations, convert $D$ to a curve $C \subset \Sigma_{d}$ in a Hirzebruch surface. If the point $P$ is of multiplicity ( $\operatorname{deg} D-3$ ), then $C$ is a trigonal curve. The fundamental group of $D$ is closely related, although not isomorphic, to that of $C$ (see Subsections 6.3 and 6.4 for details), and any information on the former can shed a light on the structure of the latter. Our principal result in this direction is Theorem 1.2.6 below. At the same time, it turns out that trigonal curves are of a certain interest on their own right (for example, as the ramification loci of elliptic surfaces), and in the framework of trigonal curves many statements relating the fundamental group and other geometric properties take more precise and complete form, see, e.g., Theorem 1.2.5.

1.2. Principal results. Given a trigonal curve $C \subset \Sigma_{d}$ (see Subsection 2.1 for the precise definitions), we will consider both the projective and affine fundamental groups

$$
\pi_{C}^{\mathrm{proj}}:=\pi_{1}\left(\Sigma_{d} \backslash(C \cup E)\right), \quad \pi_{C}^{\mathrm{afn}}:=\pi_{1}\left(\Sigma_{d} \backslash(C \cup E \cup F)\right) ;
$$

here $E \subset \Sigma_{d}$ is the exceptional section and $F \subset \Sigma_{d}$ is a fiber of the ruling that is nonsingular for $C$. The latter is a cyclic central extension of the former, and the commutants of the two groups are equal, see Corollary 3.4.5. With the usual abuse of the language, we refer to $\pi_{C}^{\text {proj }}$ and $\pi_{C}^{\text {afn }}$ as the (fundamental) groups of $C$ rather

2000 Mathematics Subject Classification. Primary: 14H30; Secondary: 14H45, 14H50.

Key words and phrases. Trigonal curve, fundamental group, dihedral covering, modular group. 
than mentioning explicitly the complement $\Sigma_{d} \backslash \ldots$ Similarly, speaking about a covering of $C$, we mean a covering of $\Sigma_{d}$ ramified at $C \cup E$.

Our main goal is substantiating the following speculation (in which Item (2) is, in fact, a statement, see Proposition 3.7.4).

\subsubsection{Speculation.}

(1) There do exist certain strict bounds on the complexity of the fundamental group of a trigonal curve; they are due to the fact that the monodromy group of such a curve is of genus zero.

(2) Any trigonal curve $C$ whose group admits a prescribed quotient $\pi_{C}^{\text {afn }} \rightarrow G$ is essentially induced from a certain universal curve with this property.

(3) As a consequence, the existence of a quotient $\pi_{C}^{\text {afn }} \rightarrow G$ as above may imply certain additional geometric properties of $C$.

(4) In particular, the existence of a quotient $\pi_{C}^{\text {afn }} \rightarrow G$ may imply the existence of a larger quotient $\pi_{C}^{\text {afn }} \rightarrow \tilde{G} \rightarrow G$.

As a first step supporting Speculation 1.2.1, we discuss the generalized dihedral quotients of $\pi_{C}^{\bullet}$. Given an abelian group $\mathcal{Q}$, the (generalized) dihedral group $\mathbb{D}(\mathcal{Q})$ is the semi-direct product $\mathcal{Q} \rtimes \mathbb{Z}_{2}$, with the factor $\mathbb{Z}_{2}$ acting on the kernel $\mathcal{Q}$ via - id. We use the standard abbreviation $\mathbb{D}_{2 n}=\mathbb{D}\left(\mathbb{Z}_{n}\right)$ for the classical dihedral groups; note that, in our notation, the index refers to the order of the group.

We are interested in the so called uniform dihedral quotients $\pi_{C}^{\bullet} \rightarrow \mathbb{D}(\mathcal{Q})$, see Definition 4.1.2. Roughly, the corresponding covering is required to have the same ramification behavior over each irreducible component of $C$. If $C$ is irreducible, then each dihedral quotient of $\pi_{C}^{\bullet}$ (respectively, each dihedral covering of $C$ ) is uniform, see Proposition 4.1.6.

1.2.2. Theorem. If the group $\pi_{C}^{\mathrm{afn}}$ of a nontrivial (see Definition 4.4.4) trigonal curve $C \subset \Sigma_{d}$ admits a uniform quotient $\mathbb{D}(\mathcal{Q})$, then $\mathcal{Q}$ is a quotient of one of the following groups:

$$
\mathbb{Z}_{2} \oplus \mathbb{Z}_{8}, \quad \mathbb{Z}_{4} \oplus \mathbb{Z}_{4}, \quad \mathbb{Z}_{2} \oplus \mathbb{Z}_{6}, \quad \mathbb{Z}_{3} \oplus \mathbb{Z}_{6}, \quad \mathbb{Z}_{9}, \quad \mathbb{Z}_{5} \oplus \mathbb{Z}_{5}, \quad \mathbb{Z}_{10}, \quad \mathbb{Z}_{7} .
$$

All quotients of the groups above do appear.

1.2.3. Theorem. A trigonal curve $C \subset \Sigma_{d}$ is reducible (respectively, splits into three components) if and only if the group $\pi_{C}^{\text {afn }}$ admits a quotient to $\mathbb{D}_{4}=\mathbb{Z}_{2} \oplus \mathbb{Z}_{2}$ (respectively, to $\left.\mathbb{D}\left(\mathbb{Z}_{2} \oplus \mathbb{Z}_{2}\right)=\mathbb{Z}_{2} \oplus \mathbb{Z}_{2} \oplus \mathbb{Z}_{2}\right)$.

1.2.4. Corollary. If an irreducible trigonal curve admits a $\mathbb{D}(\mathcal{Q})$-covering, then $\mathcal{Q}$ is a quotient of $\mathbb{Z}_{3} \oplus \mathbb{Z}_{3}, \mathbb{Z}_{9}, \mathbb{Z}_{5} \oplus \mathbb{Z}_{5}$, or $\mathbb{Z}_{7}$.

Theorem 1.2.2 and Corollary 1.2.4 are proved in Subsection 4.5; Theorem 1.2.3 is proved in Subsection 4.3. Note that Theorem 1.2.2, listing a finite set of options, is in a sharp contrast with the case of hyperelliptic curves in Hirzebruch surfaces, where each dihedral group $\mathbb{D}_{2 n}$ can appear as a uniform dihedral quotient of the fundamental group, see Remark 4.1.5 below.

One can notice a certain similarity between trigonal curves and plane sextics, where the dihedral quotients of the fundamental groups are also known, see [5]. Although the particular lists of groups differ, the prime factors appearing in their orders are the same: one has $p=3,5,7$, and, for reducible curves, $p=2$. As another similarity and, at the same time, an illustration of Statements 1.2.1(3), (4), one has the following almost literal translation of the stronger version of Oka's conjecture [9] on the Alexander polynomial of an irreducible plane sextic, see [5]. 
1.2.5. Theorem. For an irreducible trigonal curve $C \subset \Sigma_{d}$, the following four statements are equivalent:

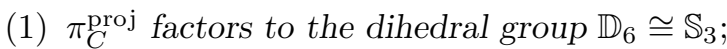

(2) $\pi_{C}^{\text {proj }}$ factors to the modular group $\Gamma \cong \mathbb{Z}_{2} * \mathbb{Z}_{3}$;

(3) $t^{2}-t+1$ divides the Alexander polynomial $\Delta_{C}(t)$, see Subsection 5.2;

(4) $C$ is of torus type, see Subsection 2.6.

This theorem is proved in Subsection 5.3, and its extension to reducible curves is discussed in Remark 5.3.1. A number of other examples illustrating 1.2.1(4) are found in Section 5, see, e.g., Corollaries 5.5.1 and 5.8.1, and a few more subtle geometric properties illustrating 1.2.1(3) (the so called $Z$-splitting sections) are discusses in Subsection 6.2.

Finally, one has the following application to the fundamental groups of plane curves, which were my original motivation for the study of trigonal curves.

1.2.6. Theorem. Let $D \subset \mathbb{P}^{2}$ be an irreducible plane curve with a singular point of multiplicity $(\operatorname{deg} D-3)$. If $D$ admits a $\mathbb{D}(\mathcal{Q})$-covering, then $\mathcal{Q}$ is a quotient of one of the groups $\mathbb{Z}_{3} \oplus \mathbb{Z}_{3}, \mathbb{Z}_{9}, \mathbb{Z}_{5} \oplus \mathbb{Z}_{5}$, or $\mathbb{Z}_{7}$.

This theorem is proved in Subsection 6.4. It is worth mentioning that the fundamental group of any irreducible plane curve $D$ with a singular point of multiplicity $(\operatorname{deg} D-1)$ is abelian, whereas for each integer $m \geqslant 1$ there is an irreducible plane curve $D$ with a singular point of multiplicity (deg $D-2)$ admitting a $\mathbb{D}_{4 m+2^{-}}$ covering.

1.3. Contents of the paper. In Section 2, we remind a few basic notions and facts related to trigonal curves in Hirzebruch surfaces. In Section 3, we discuss the braid monodromy and Zariski-van Kampen theorem computing the fundamental group, and then introduce the concept and prove the existence of universal trigonal curves related to a prescribed monodromy group or a prescribed quotient of $\pi^{\text {afn }}$. Section 4 deals with uniform dihedral quotients/coverings. The principal result here is the proof of Theorems 1.2.2 and 1.2.3. In the course of the proof, we treat the special case of isotrivial curves, which are mostly excluded from the consideration in the rest of the paper. In Section 5, we discuss the geometric properties and fundamental groups of the universal curves corresponding to uniform dihedral coverings. The results are applied to illustrate Statements 1.2.1(3) and (4) and, in particular, to prove Theorem 1.2.5. Finally, in Section 6 we discuss some further applications of the principal results: the relation to the Mordell-Weil group, $Z$ splitting sections of trigonal curves, and extensions to generalized trigonal curves and plane curves with deep singularities.

1.4. Acknowledgements. I am grateful to A. Klyachko for a number of valuable discussions concerning the modular group, and to I. Shimada, who brought to my attention paper [3].

\section{TRIgONAL CURVES}

We remind the basic notions and facts related to trigonal curves. Important for the sequel are the notions of $m$-Nagata equivalence, induced curves, and trigonal curves of torus type. 
2.1. Trigonal curves in Hirzebruch surfaces. A Hirzebruch surface $\Sigma_{d}$ is a geometrically ruled rational surface with an exceptional section $E$ of self-intersection $-d \leqslant 0$. The fibers of $\Sigma_{d}$ are the fibers of the ruling $\Sigma_{d} \rightarrow \mathbb{P}^{1}$. To avoid excessive notation, we identify fibers and their images in the base $\mathbb{P}^{1}$. The semigroup of classes of effective divisors on $\Sigma_{d}$ is freely generated by the classes $|E|$ and $|F|$, where $F$ is any fiber.

A trigonal curve is a reduced curve $C \subset \Sigma_{d}$ disjoint from the exceptional section $E \subset \Sigma_{d}$ and intersecting each fiber at three points; in other words, $C \in|3 E+3 d F|$. A trigonal curve is called simple if all its singular points are simple, i.e., those of type $\mathbf{A}_{p}, p \geqslant 1, \mathbf{D}_{q}, q \geqslant 4, \mathbf{E}_{6}, \mathbf{E}_{7}$, or $\mathbf{E}_{8}$.

A singular fiber of a trigonal curve $C \subset \Sigma_{d}$ is a fiber intersecting $C$ geometrically at fewer than three points. For the topological types of singular fibers, we use the following notation, referring to the types of the singular points of $C$ (to simplify a few statements, we sometimes extend Arnol'd's notation $\mathbf{J}, \mathbf{E}$ for the non-simple triple singular points, see [1], to the type $\mathbf{A}$ and $\mathbf{D}$ points as well):

$-\tilde{\mathbf{A}}_{0}=\tilde{\mathbf{J}}_{0,0}\left(\mathrm{I}_{0}\right)$ : a nonsingular fiber;

- $\tilde{\mathbf{A}}_{0}^{*}=\tilde{\mathbf{J}}_{0,1}\left(\mathrm{I}_{1}\right)$ : a simple vertical tangent;

- $\tilde{\mathbf{A}}_{0}^{* *}=\tilde{\mathbf{E}}_{0}$ (II): a vertical inflection tangent;

- $\tilde{\mathbf{A}}_{1}^{*}=\tilde{\mathbf{E}}_{1}$ (III): a node of $C$ with one of the branches vertical;

- $\tilde{\mathbf{A}}_{2}^{*}=\tilde{\mathbf{E}}_{2}(\mathrm{IV})$ : a cusp of $C$ with vertical tangent;

- $\tilde{\mathbf{A}}_{p}=\tilde{\mathbf{J}}_{0, p+1}\left(\mathrm{I}_{p+1}\right), p \geqslant 1$, $\tilde{\mathbf{D}}_{q}=\tilde{\mathbf{J}}_{1, q-4}\left(\mathrm{I}_{q-4}^{*}\right), q \geqslant 4$,

$\tilde{\mathbf{E}}_{6}\left(\mathrm{IV}^{*}\right), \tilde{\mathbf{E}}_{7}\left(\mathrm{III}^{*}\right), \tilde{\mathbf{E}}_{8}\left(\mathrm{II}^{*}\right)$ : a simple singular point of $C$ of the same type with the minimal possible local intersection index with the fiber;

- $\tilde{\mathbf{J}}_{r, p}, r \geqslant 2, p \geqslant 0$,

$\tilde{\mathbf{E}}_{6 r+\epsilon}, r \geqslant 2, \epsilon=0,1,2$ : a non-simple singular point of $C$ of the same type.

For the simple singular fibers we also list parenthetically Kodaira's notation for the corresponding singular fiber of the covering elliptic surface. In the case of a simple fiber, the $\mathbf{A}-\mathbf{D}-\mathbf{E}$ notation refers as well to the incidence graph of $(-2)$-curves in the corresponding elliptic fiber; this graph is an affine Dynkin diagram.

The fibers of type $\tilde{\mathbf{E}}$ (including $\tilde{\mathbf{E}}_{0}, \tilde{\mathbf{E}}_{1}$, and $\tilde{\mathbf{E}}_{2}$ ) are called exceptional.

2.2. Nagata transformations. A positive (negative) Nagata transformation is the birational transformation $\Sigma_{d} \rightarrow \Sigma_{d \pm 1}$ consisting in blowing up a point $P$ on (respectively, not on) the exceptional section $E$ and blowing down the proper transform of the fiber through $P$. An $m$-fold Nagata transformation is a sequence of $m$ Nagata transformations of the same sign over the same point of the base.

2.2.1. Definition. Two trigonal curves $C, C^{\prime}$ are called $m$-Nagata equivalent if $C^{\prime}$ is the proper transform of $C$ under a sequence of $m$-fold Nagata transformations. The special case $m=1$ is referred to as just Nagata equivalence.

Pick a fiber $F_{0}$ (fiber at infinity), consider the affine chart $\Sigma_{d} \backslash\left(E \cup F_{0}\right)$, and choose affine coordinates $(x, y)$ so that the fibers of the ruling be the vertical lines $x=$ const. In these coordinates, any trigonal curve $C$ is given by an equation of the form $\sum_{i=0}^{3} y^{i} b_{i}(x)$, where $b_{i}(x)$ is a polynomial of degree up to $d(3-i)$. In the same coordinates, a positive Nagata transformation is given by the coordinate change

$$
x=x^{\prime}, \quad y=y^{\prime} / x^{\prime}
$$


(assuming that the image of the fiber contracted is the origin $\left(x^{\prime}, y^{\prime}\right)=(0,0)$ ), and the equation of the transform of $C$ is obtained from the original equation of $C$ by the substitution and clearing the fractions. A negative Nagata transformation is given by $x=x^{\prime}, y=y^{\prime} x^{\prime}$. For the result to be disjoint from $E$, the original curve $C$ must have a triple singular point at the origin (the blow-up center); then, after the substitution, one can cancel $x^{\prime 3}$.

Under a single positive Nagata transformation, the topological type of the fiber affected changes as follows:

$$
\tilde{\mathbf{J}}_{r, p} \mapsto \tilde{\mathbf{J}}_{r+1, p}, r, p \geqslant 0, \quad \tilde{\mathbf{E}}_{6 r+\epsilon} \mapsto \tilde{\mathbf{E}}_{6(r+1)+\epsilon}, r \geqslant 0, \epsilon=0,1,2 .
$$

This statement can easily be obtained using (2.2.2) and the local normal forms. In each series, there are exactly two simple singularities, those with $r=0$ or 1 . Each series starts with its only type $\tilde{\mathbf{A}}$ singularity, corresponding to $r=0$.

2.3. The $j$-invariant. The (functional) $j$-invariant $j_{C}: \mathbb{P}^{1} \rightarrow \mathbb{P}^{1}$ of a trigonal curve $C \subset \Sigma_{d}$ is defined as the analytic continuation of the function sending a nonsingular fiber $F$ to the $j$-invariant (divided by $12^{3}$ ) of the elliptic curve covering $F$ and ramified at $F \cap(C \cup E)$. The curve $C$ is called isotrivial if $j_{C}=$ const. Such curves are easily enumerated, see Subsection 4.4.

In appropriate affine coordinates $(x, y)$ as above the curve $C$ can be given by its Weierstraß equation

$$
y^{3}+3 p(x) y+2 q(x)=0
$$

Then, the $j$-invariant is given by

$$
j_{C}(x)=\frac{p^{3}}{\Delta}, \quad \text { where } \quad \Delta(x)=p^{3}+q^{2} .
$$

Up to a constant factor, $\Delta(x)$ is the discriminant of (2.3.1) with respect to $y$.

By definition, $j_{C}$ is invariant under Nagata transformations. Any holomorphic map $j: \mathbb{P}^{1} \rightarrow \mathbb{P}^{1}$ is the $j$-invariant of a certain trigonal curve $C$, which is unique up to isomorphism and Nagata equivalence. Exceptional singular fibers of $C$ are those where $j$ takes value 0 or 1 and has ramification index $\neq 0 \bmod 3$ or $\neq 0 \bmod 2$, respectively. Singular fibers of type $\tilde{\mathbf{J}}_{r, 0}, r \geqslant 1$, are not detected by the $j$-invariant, and all other singular fibers of $C$ (those of types $\tilde{\mathbf{J}}_{r, p}, r \geqslant 0, p \geqslant 1$ ) are precisely those where $j$ takes value $\infty$. At such a fiber, the ramification index of $j$ is $p$.

Informally, the $j$-invariant $j_{C}$ determines the type $\tilde{\mathbf{J}}_{r, p}$ or $\tilde{\mathbf{E}}_{6 r+\epsilon}$ of each singular fiber of $C$ up to a choice of the integer $r \geqslant 0$. Thus, in order to select a single curve in its Nagata equivalence class, one needs to fix its type specification, i.e., select a precise type of each singular fiber and, possibly, assign types $\mathbf{J}_{r, 0}$ (not detected by the $j$-invariant) to a few generic fibers.

2.4. Maximal curves and skeletons. A non-isotrivial trigonal curve $C$ is called maximal if it has the following properties:

(1) $C$ has no singular fibers of type $\tilde{\mathbf{J}}_{r, 0}, r \geqslant 1$;

(2) $j=j_{C}$ has no critical values other than 0,1 , and $\infty$;

(3) each point in the pull-back $j^{-1}(0)$ has ramification index at most 3 ;

(4) each point in the pull-back $j^{-1}(1)$ has ramification index at most 2 . 
An important property of maximal trigonal curves is their rigidity, see [6]: any small fiberwise equisingular deformation of such a curve $C \subset \Sigma_{d}$ is isomorphic to $C$. Any maximal trigonal curve is defined over an algebraic number field.

The $j$-invariant of a maximal trigonal curve $C$ can be described by its skeleton, which is defined as the embedded bipartite graph $\mathrm{Sk}_{C}:=j_{C}^{-1}[0,1] \subset S^{2} \cong \mathbb{P}^{1}$, with the $\bullet$ - and $\circ$-vertices being the pull-backs of 0 and 1 , respectively. By definition, all $\bullet$ - (respectively, o-) vertices of $\mathrm{Sk}_{C}$ are of valency $\leqslant 3$ (respectively, $\leqslant 2$ ); in the drawings, we omit bivalent o-vertices, assuming such a vertex at the center of each edge connecting two $\bullet$-vertices. Each connected component of the complement $S^{2} \backslash \mathrm{Sk}_{C}$ is a topological disk; hence, instead of the embedding $\mathrm{Sk}_{C} \subset S^{2}$ one can regard $\mathrm{Sk}_{C}$ as a bipartite ribbon graph of genus zero.

Each skeleton Sk as above gives rise to a topological ramified covering $S^{2} \rightarrow \mathbb{P}^{1}$. By the Riemann existence theorem, the latter is realized by a holomorphic map $\mathbb{P}^{1} \rightarrow \mathbb{P}^{1}$, unique up to a Möbius transformation in the source. Hence, the skeleton $\mathrm{Sk}_{C}$ determines $j_{C}$. The type specification of a maximal trigonal curve $C$ can be regarded as a function assigning an integer $r \geqslant 0$ to each $\bullet$-vertex of valency $\leqslant 2$, each o-vertex of valency 1 , and each region of $\mathrm{Sk}_{C}$.

2.5. Induced curves. Consider a Hirzebruch surface $\Sigma:=\Sigma_{d}$ over a base $B \cong$ $\mathbb{P}^{1}$, let $B^{\prime} \cong \mathbb{P}^{1}$ be another rational curve, and let $\tilde{\jmath}: B^{\prime} \rightarrow B$ be a nonconstant holomorphic map. Then the ruled surface $\Sigma^{\prime}:=\tilde{\jmath}^{*} \Sigma$ over $B^{\prime}$ is also a Hirzebruch surface; it is isomorphic to $\Sigma_{d \cdot \operatorname{deg} \tilde{\jmath}}$. Furthermore, given a trigonal curve $C \subset \Sigma$, its divisorial pull-back $C^{\prime}:=\tilde{\jmath}^{*} C \subset \Sigma^{\prime}$ is also a trigonal curve; it is said to be induced from $C$ by $\tilde{\jmath}$ or obtained from $C$ by a rational base change.

In appropriate affine coordinates $(x, y)$ in $\Sigma$ and $\left(x^{\prime}, y^{\prime}\right)$ in $\Sigma^{\prime}$, the ramified covering $\Sigma^{\prime} \rightarrow \Sigma$ is the map

$$
\left(x^{\prime}, y^{\prime}\right) \mapsto(x, y)=\left(\frac{u\left(x^{\prime}\right)}{v\left(x^{\prime}\right)}, \frac{y^{\prime}}{v^{d}\left(x^{\prime}\right)}\right)
$$

here, $x$ and $x^{\prime}$ are affine parameters in $B$ and $B^{\prime}$, respectively, and $\tilde{\jmath}$ is given by the reduced fraction $\tilde{\jmath}\left(x^{\prime}\right)=u\left(x^{\prime}\right) / v\left(x^{\prime}\right)$. An equation for $C^{\prime}$ is obtained from that for $C$ by substituting (2.5.1) and clearing denominators.

Locally, the substitution is given by $\left(x^{\prime}, y^{\prime}\right) \mapsto(x, y)=\left(x^{\prime m}, y^{\prime}\right)$, where $m$ is the ramification index of $\tilde{\jmath}$ at $x^{\prime}=0$, and, using local normal forms, one can easily find the types of the singular fibers of $C^{\prime}$ in terms of those of $C$. Next lemma characterizes Kodaira type I fibers, i.e., types $\tilde{\mathbf{A}}_{0}^{*}$ and $\tilde{\mathbf{A}}_{p}, p \geqslant 1$.

2.5.2. Lemma. If a trigonal curve $C$ has Kodaira type I singular fibers only, then any curve $C^{\prime}$ induced from $C$ by a rational base change $\tilde{\jmath}$ is simple; in fact, $C^{\prime}$ also has Kodaira type I singular fibers only.

Proof. The proof is a simple computation using local normal forms, as explained above. Assume that $\tilde{\jmath}$ sends $F^{\prime}=\left\{x^{\prime}=0\right\}$ to $F=\{x=0\}$ and has ramification index $m$ at $F^{\prime}$. If $F$ is of type $\mathrm{I}_{p+1}, p \geqslant 0$, then $F^{\prime}$ is of type $\mathrm{I}_{m(p+1)}$. If $F$ is of any other type, its local normal form is $y^{3}+x a(x, y)$ and, for any $m>6$, the induced fiber $F^{\prime}$ is not simple.

2.6. Trigonal curves of torus type. A trigonal curve $C$ in an even Hirzebruch surface $\Sigma_{2 k}$ is said to be of torus type if there are sections $f_{i}$ of $\mathcal{O}(E+i k F)$, 
$i=0,2,3$, such that $C$ is the zero set of the section $f_{2}^{3}+f_{0} f_{3}^{2}$. Informally, in affine coordinates $(x, y)$ with $E=\{y=\infty\}$, the equation of $C$ has the form

$$
\left[y+a_{2}(x)\right]^{3}+\left[a_{1}(x) y+a_{3}(x)\right]^{2}=0
$$

for some polynomials $a_{i}(x)$ of degree up to $k i, i=1,2,3$. Each representation of the equation of $C$ in this form (up to an obvious equivalence) is called a torus structure on $C$.

2.6.2. Lemma. Torus structures are preserved under rational base changes and 2-fold Nagata transformations.

Proof. Consider a rational base change $\mathbb{P}^{1} \rightarrow \mathbb{P}^{1}$ given by $x=\tilde{\jmath}\left(x^{\prime}\right):=u\left(x^{\prime}\right) / v\left(x^{\prime}\right)$, $y=y^{\prime} / v^{2 k}\left(x^{\prime}\right)$, see (2.5.1). Substituting to (2.6.1) and clearing denominators, one obtains

$$
\left[y^{\prime}+a_{2}^{\prime}\left(x^{\prime}\right)\right]^{3}+\left[a_{1}^{\prime}\left(x^{\prime}\right) y^{\prime}+a_{3}^{\prime}\left(x^{\prime}\right)\right]^{2}=0,
$$

where $a_{i}^{\prime}\left(x^{\prime}\right)=a_{i}(u / v) v^{k i}\left(x^{\prime}\right), i=1,2,3$, i.e., again an equation of the form (2.6.1).

Now, consider a positive 2-fold Nagata transformation $x=x^{\prime}, y=y^{\prime} / x^{\prime 2}$, see (2.2.2); we can assume that the fiber contracted is over $x=0$. Substituting and clearing denominators, one obtains (2.6.3) with $a_{i}^{\prime}\left(x^{\prime}\right)=a_{i}\left(x^{\prime}\right) x^{\prime i}, i=1,2,3$.

Finally, consider a negative 2-fold Nagata transformation; in appropriate affine coordinates it is given by $x=x^{\prime}, y=y^{\prime} x^{\prime 2}$. For the transform to be disjoint from the exceptional section, the singularity of the original curve at the origin must be adjacent to $\mathbf{J}_{2,0}$, i.e., all terms $x^{\alpha} y^{\beta}, 2 \alpha+\beta<6$, of the original equation must vanish. Evaluating at $x=0$, one can easily see that $a_{1}(0)=a_{2}(0)=a_{3}(0)=0$, and then, step by step, one can conclude that $a_{i}(x)=x^{i} a_{i}^{\prime}(x)$ for some polynomials $a_{i}^{\prime}(x), i=1,2,3$. Substituting and cancelling $x^{\prime 6}$, one arrives at (2.6.3).

2.6.4. Remark. If $C$ is the proper transform of a curve $C^{\prime}$ of torus type under a negative Nagata transformation contracting a fiber $F$, then the divisorial transform of $C^{\prime}$ inherits a torus structure: one has $C+s F=\left\{f_{2}^{3}+f_{0} f_{3}^{2}=0\right\}$ for some $s \geqslant 0$. What is shown in the proof above is that, if after a 2 -fold transformation $C$ is still disjoint from $E$, then $s=6$ and the curves $\left\{f_{2}=0\right\}$ and $\left\{f_{3}=0\right\}$ contain $F$ with multiplicity at least 2 and 3 , respectively, so that $6 F$ can be factored out.

2.6.5. Lemma. If a trigonal curve $C \subset \Sigma_{2 k}$ is of torus type, there exists a triple covering $X \rightarrow \Sigma_{2 k}$ ramified at $C \cup E$ with the full monodromy group $\mathbb{S}_{3}$.

Proof. In affine coordinates $(x, y, z)$ in $\mathbb{C}^{2} \times \mathbb{C}$, the covering surface $X$ is given by $z^{3}+3\left(y+a_{2}\right)+2\left(a_{1} y+a_{3}\right)=0$, see (2.6.1). More formally, $X$ is the normalization of the triple section of $\mathcal{O}(E+k F)$ given by $z^{3}+3 f_{0} f_{2} z+2 f_{0}^{2} f_{3}=0$. Restricting to a generic fiber $x=$ const, one obtains a covering $C_{x} \rightarrow \mathbb{P}^{1}$, where $C_{x} \subset \Sigma_{1}$ is a trigonal curve with the set of singular fibers $\tilde{\mathbf{A}}_{1}^{*} \oplus 3 \tilde{\mathbf{A}}_{0}^{*}$. All such curves are deformation equivalent (essentially, they are nodal plane cubics projected from a generic point in the tangent to one of the branches at the node), and their monodromy groups are easily shown to equal $\mathbb{S}_{3}$, see Remark 4.3 .1 below for details.

2.6.6. Remark. If $C$ is irreducible, the converse of Lemma 2.6 .5 also holds: it is given by Theorem 1.2.5, proved in Subsection 5.3. 


\section{THE BRAID MONODROMY}

In this section, we define the braid monodromy and its various reductions, cite Zariski-van Kampen theorem and its implications for the particular case of trigonal curves, introduce the concept of universal curves, and prove their existence.

3.1. Groups to be considered. Let $\mathcal{H}=\mathbb{Z} a \oplus \mathbb{Z} b$ be a rank 2 free abelian group with the skew-symmetric bilinear form $\bigwedge^{2} \mathcal{H} \rightarrow \mathbb{Z}$ given by $a \cdot b=1$. We fix the notation $\mathcal{H}, a, b$ throughout the paper and define $\tilde{\Gamma}:=S L(2, \mathbb{Z})$ as the group $\operatorname{Sp} \mathcal{H}$ of symplectic auto-isometries of $\mathcal{H}$; it is generated by the isometries $\mathbb{X}, \mathbb{Y}: \mathcal{H} \rightarrow \mathcal{H}$ given (in the basis $\{a, b\}$ above) by the matrices

$$
\mathbb{X}=\left[\begin{array}{ll}
-1 & 1 \\
-1 & 0
\end{array}\right], \quad \mathbb{Y}=\left[\begin{array}{rr}
0 & -1 \\
1 & 0
\end{array}\right]
$$

One has $\mathbb{X}^{3}=\mathrm{id}$ and $\mathbb{Y}^{2}=-\mathrm{id}$. The modular group $\Gamma:=\operatorname{PSL}(2, \mathbb{Z})$ is the quotient $\tilde{\Gamma} / \pm$ id. We retain the notation $\mathbb{X}, \mathbb{Y}$ for the generators of $\Gamma$. One has

$$
\Gamma=\left\langle\mathbb{X} \mid \mathbb{X}^{3}=1\right\rangle *\left\langle\mathbb{Y} \mid \mathbb{Y}^{2}=1\right\rangle \cong \mathbb{Z}_{3} * \mathbb{Z}_{2}
$$

The braid group $\mathbb{B}_{3}$ is the group

$$
\mathbb{B}_{3}=\left\langle\sigma_{1}, \sigma_{2} \mid \sigma_{1} \sigma_{2} \sigma_{1}=\sigma_{2} \sigma_{1} \sigma_{2}\right\rangle=\left\langle u, v \mid u^{3}=v^{2}\right\rangle,
$$

where $u=\sigma_{2} \sigma_{1}$ and $v=\sigma_{2} \sigma_{1}^{2}$. The center $Z\left(\mathbb{B}_{3}\right)$ is the infinite cyclic subgroup generated by $u^{3}=v^{2}$, and the quotient $\mathbb{B}_{3} / Z\left(\mathbb{B}_{3}\right)$ is isomorphic to $\Gamma$. We define the epimorphism $\mathbb{B}_{3} \rightarrow \tilde{\Gamma}$ (and further to $\Gamma$ ) via

$$
\sigma_{1} \mapsto \mathbb{X} \mathbb{Y}=\left[\begin{array}{ll}
1 & 1 \\
0 & 1
\end{array}\right], \quad \sigma_{2} \mapsto \mathbb{X}^{2} \mathbb{Y} \mathbb{X}^{-1}=\left[\begin{array}{rr}
0 & 1 \\
-1 & 2
\end{array}\right]
$$

Then $u \mapsto-\mathbb{X}^{-1}$ and $v \mapsto-\mathbb{Y}$. This unusual choice of generators is explained in Remark 3.3.4 below.

The braid group $\mathbb{B}_{3}$ acts on the free group $\left\langle\alpha_{1}, \alpha_{2}, \alpha_{3}\right\rangle$ via

$$
\sigma_{1}: \alpha_{1} \mapsto \alpha_{1} \alpha_{2} \alpha_{1}^{-1}, \quad \alpha_{2} \mapsto \alpha_{1} ; \quad \sigma_{2}: \alpha_{2} \mapsto \alpha_{2} \alpha_{3} \alpha_{2}^{-1}, \quad \alpha_{3} \mapsto \alpha_{2} .
$$

According to E. Artin [2], $\mathbb{B}_{3}$ can be identified with the group of automorphisms of $\left\langle\alpha_{1}, \alpha_{2}, \alpha_{3}\right\rangle$ taking each generator to a conjugate of a generator and preserving the product $\rho:=\alpha_{1} \alpha_{2} \alpha_{3}$. In what follows, we fix the notation $\mathfrak{F}$ for the free group $\left\langle\alpha_{1}, \alpha_{2}, \alpha_{3}\right\rangle$ supplied with this $\mathbb{B}_{3}$-action. We do not distinguish between the original basis $\left\{\alpha_{1}, \alpha_{2}, \alpha_{3}\right\}$ and any other basis in its $\mathbb{B}_{3}$-orbit; any basis in the latter orbit is called geometric. We also reserve the notation $\rho$ for the product $\alpha_{1} \alpha_{2} \alpha_{3}$.

The group $\mathfrak{F}$ is also equipped with a distinguished homomorphism $\operatorname{deg}: \mathfrak{F} \rightarrow \mathbb{Z}$, $\alpha_{i} \mapsto 1$, which does not depend on the choice of a geometric basis.

Any finite index subgroup $G \subset \mathbb{B}_{3}$ must intersect the center $Z\left(\mathbb{B}_{3}\right)$. We define the depth $\operatorname{dp} G$ as the minimal positive integer $m$ such that $\left(\sigma_{2} \sigma_{1}\right)^{3 m} \in G$.

We are mainly interested in subgroups of $\mathbb{B}_{3}, \tilde{\Gamma}$, or $\Gamma$ up to conjugation. Given two subgroups $H, H^{\prime} \subset G$, we say that $H^{\prime}$ is subconjugate to $H$, notation $H^{\prime} \prec H$, if $H^{\prime}$ is conjugate in $G$ to a subgroup of $H$. 
3.2. Skeletons and genus. Given a finite index subgroup $G \subset \Gamma$, the quotient $\Gamma / G$ can be given a natural structure of a bipartite ribbon graph; it is denoted by $\mathrm{Sk}_{G}$ and called the skeleton of $G$. The set of edges of $\mathrm{Sk}_{G}$ is $\Gamma / G$. This set has a canonical left $\Gamma$-action, and we define the $\bullet$ - and o-vertices of $\mathrm{Sk}_{G}$ as the orbits of $\mathbb{X}$ and $\mathbb{Y}$, respectively. The cyclic order (the ribbon graph structure) at a trivalent --vertex is given by $\mathbb{X}^{-1}$; all other vertices are at most bivalent, and the cyclic order is unique. Alternatively, there is a natural $\Gamma$-action on the infinite Farey tree $\mathrm{F}$ (the only bipartite tree with all $\bullet$-vertices of valency 3 and all o-vertices of valency 2 ), and one can define $\mathrm{Sk}_{G}$ as $\mathrm{F} / G$. For more details and all proofs, see [7].

By definition, the valency of each - (respectively, o-) vertex of $\mathrm{Sk}_{G}$ is divisible by 3 (respectively, 2). In the drawings, we omit bivalent o-vertices, assuming that such a vertex is to be inserted at the middle of each edge connecting two $\bullet$-vertices. Conversely, the set of edges of any bipartite ribbon graph Sk satisfying the above valency condition admits a natural $\Gamma$-action, and the stabilizer of a fixed edge of Sk is a certain finite index subgroup $G \subset \Gamma$. One has $\mathrm{Sk} \cong \mathrm{Sk}_{G}$.

3.2.1. Remark. Both skeletons of maximal trigonal curves, see Subsection 2.4, and skeletons of subgroups of $\Gamma$ defined above are bipartite ribbon graph with all - vertices of valency $\leqslant 3$ and all o-vertices of valency $\leqslant 2$; in the figures, we use the same convention about bivalent o-vertices. Note though that there is a certain difference between the two classes: skeletons of trigonal curves are required to be of genus zero (due to the fact that we consider curves over a rational base only), whereas skeletons of subgroups are not allowed to have bivalent •-vertices. Still, the two notions are closely related, $c f$. Remark 3.3.5 below.

The monovalent vertices of $\mathrm{Sk}_{G}$ are in a one-to-one correspondence with the torsion elements of $G$, and its regions, i.e., minimal left turn cycles, correspond to the conjugacy classes of indivisible parabolic elements of $G$. If $G$ is torsion free, equivalently, if $\mathrm{Sk}_{G}$ has no monovalent vertices, there is a canonical isomorphism $G=\pi_{1}\left(\mathrm{Sk}_{G}\right)$. In general, $G$ is isomorphic to an appropriately defined orbifold fundamental group $\pi_{1}^{\text {orb }}\left(\mathrm{Sk}_{G}\right)$.

3.2.2. Definition. If $[\Gamma: G]<\infty$, the genus of the minimal surface supporting the skeleton $\mathrm{Sk}_{G}$ is called the genus of $G$. The genus of a finite index subgroup of $\tilde{\Gamma}$ or $\mathbb{B}_{3}$ is defined as the genus of its image in $\Gamma$.

Genus is nonnegative, invariant under conjugation, and monotonous: one has $\operatorname{genus}\left(G^{\prime}\right) \geqslant \operatorname{genus}(G) \geqslant 0$ whenever $G^{\prime} \prec G$.

Definition 3.2.2 is equivalent to the classical definition of genus, see Remark 3.5.1 below. It is worth emphasizing that, when speaking about a subgroup $G \subset \mathbb{B}_{3}$ of genus zero, we always assume that $G$ is of finite index, i.e., $G \cap Z\left(\mathbb{B}_{3}\right) \neq\{1\}$.

3.3. Proper sections and braid monodromy. In the exposition below, we follow the approach of [4], which makes certain choices in the definition of braid monodromy more canonical. We refer to [4] for most proofs, which are omitted.

Fix a Hirzebruch surface $\Sigma_{d}$ and a trigonal curve $C \subset \Sigma_{d}$. The term 'section' stands for a continuous section of (a restriction of) the fibration $p: \Sigma_{d} \rightarrow \mathbb{P}^{1}$. For any fiber $F$ of $\Sigma_{d}$, the complement $F^{\circ}:=F \backslash E$ is an affine space over $\mathbb{C}$. Hence, one can speak about the convex hull of a subset of $F^{\circ}$. For a subset $S \subset \Sigma_{d} \backslash E$, we denote by $\operatorname{conv}_{F} S$ the convex hull of $S \cap F^{\circ}$ in $F^{\circ}$ and let conv $S$ be the union of $\operatorname{conv}_{F} S$ over all fibers $F$. 
3.3.1. Definition. Let $\Delta \subset \mathbb{P}^{1}$ be a closed topological disk. A section $s: \Delta \rightarrow \Sigma_{k}$ of $p$ is called proper if its image is disjoint from both $E$ and $\operatorname{conv} C$.

Any disk $\Delta \subset \mathbb{P}^{1}$ admits a proper section $s: \Delta \rightarrow \Sigma_{k}$, unique up to homotopy in the class of proper sections.

Fix a disk $\Delta \subset \mathbb{P}^{1}$ and let $F_{1}, \ldots, F_{r} \in \Delta$ be all singular and, possibly, some nonsingular fibers of $C$ that belong to $\Delta$. Assume that all these fibers are in the interior of $\Delta$. Let $\Delta^{\circ}=\Delta \backslash\left\{F_{1}, \ldots, F_{r}\right\}$ and fix a reference fiber $F \in \Delta^{\circ}$. Then, given a proper section $s$, one can define the group $\pi_{F}:=\pi_{1}\left(F^{\circ} \backslash C, s(F)\right)$ and the braid monodromy, which is the anti-homomorphism $\mathfrak{m}: \pi_{1}\left(\Delta^{\circ}, F\right) \rightarrow$ Aut $\pi_{F}$ sending a loop $\gamma$ to the automorphism obtained by dragging $F$ along $\gamma$ and keeping the reference point in $s$.

3.3.2. Definition. Let $D$ be an oriented punctured disk, and let $b \in \partial D$. A geometric basis in $D$ is a basis $\left\{\gamma_{1}, \ldots, \gamma_{r}\right\}$ for the free group $\pi_{1}(D, b)$ formed by the classes of positively oriented lassoes about the punctures, pairwise disjoint except at the common reference point $b$ and such that $\gamma_{1} \ldots \gamma_{r}=[\partial D]$.

Shrink the reference fiber $F$ to a closed disk containing $\operatorname{conv}_{F} C$ in its interior and $s(F)$ in its boundary. Pick a geometric basis for $\pi_{F}$ and identify it with a geometric basis $\left\{\alpha_{1}, \alpha_{2}, \alpha_{3}\right\}$ for $\mathfrak{F}$, establishing an isomorphism $\pi_{F} \cong \mathfrak{F}$. Due to Artin's theorem [2], under this isomorphism the braid monodromy $\mathfrak{m}$ takes values in the braid group $\mathbb{B}_{3} \subset$ Aut $\mathfrak{F}$, which explains the term. The $\mathbb{B}_{3}$-valued braid monodromy $\mathfrak{m}$ thus defined is independent of the choice of a proper section, and another choice of the geometric bases used for the identification $\pi_{F}=\mathfrak{F}$ results in the global conjugation by a fixed braid $\beta \in \mathbb{B}_{3}$, i.e., in the map $\gamma \mapsto \beta^{-1} \mathfrak{m}(\gamma) \beta$.

3.3.3. Lemma. Assume that the disk $\Delta$ contains all singular fibers of $C$. Then, in any geometric basis $\left\{\gamma_{1}, \ldots, \gamma_{r}\right\}$ in $\Delta^{\circ}$, the so called monodromy at infinity $\mathfrak{m}\left(\gamma_{1} \ldots \gamma_{r}\right)=\mathfrak{m}[\partial \Delta]$ equals $\left(\sigma_{2} \sigma_{1}\right)^{3 d}$; it is the conjugation by $\rho^{d}$.

In what follows, we will take for the disk $\Delta$ the complement of a small regular neighborhood of a nonsingular fiber $F_{0} \in \mathbb{P}^{1}$. Due to Lemma 3.3.3, the braid monodromy over $\Delta$ factors to an anti-homomorphism $\mathfrak{m}: \pi_{1}\left(B^{\circ}\right) \rightarrow \mathbb{B}_{3} /\left(\sigma_{2} \sigma_{1}\right)^{3 d}$, where $B^{\circ}=\mathbb{P}^{1} \backslash \bigcup_{i=1}^{r} F_{r}$. This map is independent of the choice of $F_{0}$. Its image $\mathfrak{I m}_{C}\left(\mathbb{B}_{3}\right)$, regarded as a subgroup of $\mathbb{B}_{3}$, is called the monodromy group of $C$; it is defined by $C$ up to conjugation. We will also consider the reductions of $\mathfrak{m}$ to the groups $\tilde{\Gamma}$ and $\Gamma$; their images are denoted by $\mathfrak{I m}_{C}(\tilde{\Gamma})$ and $\mathfrak{I m}_{C}(\Gamma)$, respectively.

3.3.4. Remark. The $\Gamma$-valued braid monodromy $\mathfrak{m}: \pi_{1}\left(B^{\circ}\right) \rightarrow \Gamma$ is always well defined. For a non-isotrivial trigonal curve $C$, it can be expressed in terms of its $j$-invariant $j_{C}$. Let $B_{\Gamma}$ be the Riemann sphere $\mathbb{C} \cup\{\infty\} \cong \mathbb{P}^{1}$, and denote $B_{\Gamma}^{\circ}=B_{\Gamma} \backslash\{0,1, \infty\}$. The identification of $B_{\Gamma}$ with the modular curve $\Gamma \backslash \mathfrak{H}^{*}$, see, e.g., [16], gives rise to a canonical principal $\Gamma$-bundle over $B_{\Gamma}^{\circ}$, which defines the monodromy (anti-)representation $\mathfrak{m}_{\Gamma}: B_{\Gamma} \rightarrow \Gamma$. Then, up to global conjugation, the $\Gamma$-valued braid monodromy of a curve $C$ is the composition $\mathfrak{m}_{\Gamma} \circ\left(j_{C}\right)_{*}$. This fact is well known for the homological invariant of an elliptic surface; the relation between the modular representation and the braid monodromy of a trigonal curve can be established, e.g., using the computation in [6].

Accidentally, it is this reduction that motivates our not quite usual choice of the epimorphism $\mathbb{B}_{3} \rightarrow \tilde{\Gamma}$ fixed in Subsection 3.1: it is consistent with a canonical basis $\left\{\alpha_{1}, \alpha_{2}, \alpha_{3}\right\}$ for $\pi_{F}$ constructed in [6] and the basis $a=\alpha_{2} \alpha_{1}, b=\alpha_{1} \alpha_{3}$ for the 
group $\mathcal{H}$ regarded as the 1-homology of the double covering of $F$, see Subsection 4.1 and (6.1.2) below.

3.3.5. Remark. It follows, in particular, that the skeleton of a maximal trigonal curve without type $\tilde{\mathbf{E}}_{6 r+2}, r \geqslant 0$, singular fibers, i.e., without bivalent •-vertices, can be identified with the skeleton of its monodromy group, see [7]. Hence, such a curve is determined by the conjugacy class of its $\Gamma$-valued monodromy group up to Nagata equivalence. Furthermore, each genus zero subgroup of $\Gamma$ determines a unique Nagata equivalence class of maximal trigonal curves; the curve can be made unique up to isomorphism if all singular fibers are required to be of type $\tilde{\mathbf{A}}$.

3.3.6. Lemma. In the notation of Lemma 3.3.3, assume that $C^{\prime}$ is obtained from $C$ by a positive Nagata transformation at a fiber $F_{i}$, and let $\mathfrak{m}^{\prime}$ be the braid monodromy of $C^{\prime}$. Then $\mathfrak{m}^{\prime}\left(\gamma_{j}\right)=\mathfrak{m}\left(\gamma_{j}\right)$ for $j \neq i$, and $\mathfrak{m}^{\prime}\left(\gamma_{i}\right)=\mathfrak{m}\left(\gamma_{i}\right)\left(\sigma_{2} \sigma_{1}\right)^{3}$.

Proof. The statement follows from (2.2.2): the monodromy of $C^{\prime}$ about $F_{i}$ differs from that of $C$ by an extra full twist, i.e., $\left(\sigma_{2} \sigma_{1}\right)^{3}$ or the conjugation by $\rho$.

3.3.7. Remark. According to Lemma 3.3.6, the choice of a type specification selecting a curve within its Nagata equivalence class is equivalent to the choice of a lift to $\mathbb{B}_{3}$ of the $\Gamma$-valued braid monodromy determined by the $j$-invariant.

3.3.8. Lemma. Assume that a trigonal curve $C^{\prime}$ is induced from $C$ by a rational base change $\tilde{\jmath}: B^{\prime} \rightarrow B$. Then $\mathfrak{I m}_{C^{\prime}}\left(\mathbb{B}_{3}\right) \prec \mathfrak{I m}_{C}\left(\mathbb{B}_{3}\right)$.

Proof. The data $\Delta^{\prime}, s^{\prime}, F^{\prime}$ necessary to define the monodromy $\mathfrak{m}^{\prime}$ of $C^{\prime}$ can be pulled back from those for $C$; namely, let $\Delta^{\prime}=\tilde{j}^{-1}(\Delta)$ (formally, with a few extra cuts to make it a disk) and $s^{\prime}=\tilde{j}^{*} s$ and take for $F^{\prime}$ any fiber in the pull-back of $F$. Then obviously $\mathfrak{m}^{\prime}=\mathfrak{m} \circ \tilde{\jmath}_{*}$, and the statement follows.

3.4. The Zariski-van Kampen theorem. Consider a trigonal curve $C \subset \Sigma_{d}$. Keeping the notation of Subsection 3.3, fix a nonsingular fiber $F_{0}$ of $C$, denote by $F_{1}, \ldots, F_{r}$ all its singular (and possibly some nonsingular) fibers, identify the group of the reference fiber with $\mathfrak{F}$, and let $\mathfrak{m}: \pi_{1}\left(\Delta^{\circ}\right) \rightarrow \mathbb{B}_{3}$ be the resulting braid monodromy. Fix also a geometric basis $\left\{\gamma_{1}, \ldots, \gamma_{r}\right\}$ in the punctured disk $\Delta^{\circ}$ and let $\gamma_{0}=\left(\gamma_{1} \ldots \gamma_{r}\right)^{-1}$ : this class is represented by a small loop about $F_{0}$.

The following theorem is essentially contained in [11].

3.4.1. Theorem. In the notation above, one has

$$
\begin{aligned}
& \pi_{1}\left(\Sigma_{d} \backslash\left(C \cup E \cup \bigcup_{i=0}^{r} F_{r}\right)\right)=\left\langle\alpha_{1}, \alpha_{2}, \alpha_{3}, \tilde{\gamma}_{0}, \tilde{\gamma}_{1}, \ldots, \tilde{\gamma}_{r}\right| \\
& \left.\quad \tilde{\gamma}_{i}^{-1} \alpha_{j} \tilde{\gamma}_{i}=\mathfrak{m}_{i}\left(\alpha_{j}\right), i=1, \ldots, r, j=1,2,3, \tilde{\gamma}_{0} \tilde{\gamma}_{1} \ldots \tilde{\gamma}_{r} \rho^{d}=1\right\rangle,
\end{aligned}
$$

where $\mathfrak{m}_{i}=\mathfrak{m}\left(\gamma_{i}\right), i=1, \ldots, r$, and $\tilde{\gamma}_{i}$ is a certain lift of $\gamma_{i}, i=0, \ldots, r$.

Patching back in a fiber $F_{i}, i=0, \ldots, r$, results in an extra relation $\tilde{\gamma}_{i}=1$. Hence, one has the following corollary.

3.4.2. Corollary. One has

$$
\begin{aligned}
\pi_{C}^{\mathrm{afn}} & =\left\langle\alpha_{1}, \alpha_{2}, \alpha_{3} \mid \mathfrak{m}\left(\gamma_{i}\right)=\mathrm{id}, i=1, \ldots, r\right\rangle, \\
\pi_{C}^{\text {proj }} & =\left\langle\alpha_{1}, \alpha_{2}, \alpha_{3} \mid \mathfrak{m}\left(\gamma_{i}\right)=\mathrm{id}, i=1, \ldots, r, \rho^{d}=1\right\rangle,
\end{aligned}
$$

where each braid relation $\mathfrak{m}\left(\gamma_{i}\right)=$ id should be regarded as a triple of relations $\mathfrak{m}\left(\gamma_{i}\right)\left(\alpha_{j}\right)=\alpha_{j}, j=1,2,3$, or, equivalently, as a set of relations $\mathfrak{m}\left(\gamma_{i}\right)(\alpha)=\alpha$ for each $\alpha \in\left\langle\alpha_{1}, \alpha_{2}, \alpha_{3}\right\rangle$. 
3.4.3. Definition. The presentations of $\pi_{C}^{\text {afn }}$ and $\pi_{C}^{\text {proj }}$ given by Corollary 3.4 .2 are called geometric. More precisely, a geometric presentation is an epimorphism $\mathfrak{F} \rightarrow \pi_{C}^{\text {afn }}$ or $\mathfrak{F} \rightarrow \pi_{C}^{\text {proj }}$ obtained by identifying a geometric basis in a nonsingular fiber $F_{0}$ and a geometric basis of $\mathfrak{F}$.

3.4.4. Remark. In other words, Corollary 3.4 .2 states that $\pi_{C}^{\text {afn }}$ is the quotient of $\mathfrak{F}$ by the minimal normal subgroup containing $g(\alpha) \alpha^{-1}$ for all $g \in \mathfrak{I m}_{C}\left(\mathbb{B}_{3}\right)$ and $\alpha \in \mathfrak{F}$, and $\pi_{C}^{\mathrm{proj}}$ is the further quotient of $\pi_{C}^{\mathrm{afn}}$ by the normal subgroup generated by $\rho^{d} \in \pi_{C}^{\mathrm{afn}}$.

3.4.5. Corollary. Given a trigonal curve $C \subset \Sigma_{d}$, the canonical epimorphism $\pi_{C}^{\text {afn }} \rightarrow \pi_{C}^{\text {proj }}$ is a central extension by the infinite cyclic subgroup generated by $\rho^{d} \in \pi_{C}^{\mathrm{afn}}$. This epimorphism induces an isomorphism $\left[\pi_{C}^{\mathrm{afn}}, \pi_{C}^{\mathrm{afn}}\right]=\left[\pi_{C}^{\mathrm{proj}}, \pi_{C}^{\mathrm{proj}}\right]$. In particular, the group $\pi_{C}^{\text {afn }}$ is abelian if and only if so is $\pi_{C}^{\text {proj }}$.

Proof. The element $\rho^{d}$ that normally generates the kernel, see Remark 3.4.4, is central due to Lemma 3.3.3. It follows from Corollary 3.4.2 that $\rho^{d}$ is mapped to an infinite order element of the abelianization $\pi_{C}^{\mathrm{afn}} /\left[\pi_{C}^{\mathrm{afn}}, \pi_{C}^{\mathrm{afn}}\right]$. (In particular, $\rho^{d}$ itself is of infinite order and the kernel $\left\langle\rho^{d}\right\rangle$ is infinite.) Hence, $\left\langle\rho^{d}\right\rangle \cap\left[\pi_{C}^{\mathrm{afn}}, \pi_{C}^{\mathrm{afn}}\right]=\{1\}$, and the induced homomorphism of the commutants is one-to-one. Since it is also onto (as induced by an epimorphism), it is an isomorphism.

3.4.6. Corollary. If two trigonal curves $C$ and $C^{\prime}$ are $m$-Nagata equivalent, the quotients of the groups $\pi_{C}^{\text {afn }}$ and $\pi_{C^{\prime}}^{\text {afn }}$ by the commutator subgroups $\left[\pi_{\bullet}^{\text {afn }},\left\langle\rho^{m}\right\rangle\right]$ are canonically isomorphic.

Proof. Computing the groups modulo the extra relations $\left[\alpha, \rho^{m}\right]=1, \alpha \in \mathfrak{F}$, one can reduce the braid monodromy to $\mathbb{B}_{3} /\left(\sigma_{2} \sigma_{1}\right)^{3 m}$. According to Lemma 3.3.6, the two reductions coincide.

3.4.7. Lemma. Assume that a trigonal curve $C^{\prime}$ is induced from a curve $C$ by a rational base change $\tilde{\jmath}: B^{\prime} \rightarrow B$. Then $\tilde{\jmath}$ induces epimorphisms $\pi_{C^{\prime}}^{\text {afn }} \rightarrow \pi_{C}^{\text {afn }}$ and $\pi_{C^{\prime}}^{\text {proj }} \rightarrow \pi_{C}^{\text {proj }}$ compatible with geometric presentations of the groups.

Proof. The statement follows from Corollary 3.4.2 (see also Remark 3.4.4) and Lemma 3.3.8.

3.5. Universal curves. Recall that there is a canonical faithful discrete action of the modular group $\Gamma$ on the upper half plane $\mathfrak{H}:=\{z \in \mathbb{C} \mid \operatorname{Im} z>0\}$, so that $\Gamma \backslash \mathfrak{H}=\mathbb{C}$. Let $\mathfrak{H}^{\circ}$ be $\mathfrak{H}$ with the orbits of $i$ and $\exp (2 \pi i / 3)$ removed; the action is free on $\mathfrak{H}^{\circ}$. For a subgroup $G \subset \Gamma$, denote $B_{G}^{\circ}=G \backslash \mathfrak{H}^{\circ}$, and let $j_{G}: B_{G}^{\circ} \rightarrow B_{\Gamma}^{\circ}=$ $\mathbb{C} \backslash\{0,1\}$ be the projection. One obviously has $\operatorname{deg} j_{G}=[\Gamma: G]$ and the image $\left(j_{C}\right)_{*} \pi_{1}\left(B_{G}^{\circ}\right) \subset \pi_{1}\left(B_{\Gamma}^{\circ}\right)$ is the pull-back of $G$ under the modular representation, see Remark 3.3.4. If $G$ is of finite index, the Riemann surface $B_{G}^{\circ}$ can be compactified to a ramified covering $j_{G}: B_{G} \rightarrow B_{\Gamma}=\mathbb{P}^{1}$. In this case, one has $B_{G}=G \backslash \mathfrak{H}^{*}$, where $\mathfrak{H}^{*}=\mathfrak{H} \cup \mathbb{Q} \cup\{\infty\}$, see, e.g., [16].

3.5.1. Remark. The skeleton $\mathrm{Sk}_{G}$ of a finite index subgroup $G \subset \Gamma$ can be identified with the ribbon graph $j_{G}^{-1}[0,1] \subset B_{G}$, the $\bullet$ - and o-vertices being the pull-backs of 0 and 1 , respectively. Furthermore, since $j_{G}$ is ramified over 0,1 , and $\infty$ only, $B_{G}$ is a minimal surface supporting $\mathrm{Sk}_{G}$ and the genus of $G$ given by Definition 3.2.2 equals the genus of $B_{G}$, which is the classical definition. 
3.5.2. Lemma. Let $C$ be a non-isotrivial trigonal curve over $B$, and assume that $\mathfrak{I m}_{C}(\Gamma) \prec G$ for some subgroup $G \subset \Gamma$. Then the $j$-invariant $j_{C}: B \rightarrow \mathbb{P}^{1}$ factors through $j_{G}: B_{G} \rightarrow B_{\Gamma}=\mathbb{P}^{1}$.

Proof. Assume that $\mathfrak{I m}_{C}(\Gamma) \subset G$ and consider the (ramified) coverings $j_{C}: B \rightarrow \mathbb{P}^{1}$ and $j_{G}: B_{G}^{\circ} \rightarrow B_{\Gamma}^{\circ}=\mathbb{P}^{1} \backslash\{0,1, \infty\}$. Denote by $B_{\Gamma}^{\circ \circ}$ the base $B_{\Gamma}^{\circ}$ with the critical values of $j_{C}$ removed, and let $B_{G}^{\circ \circ}=j_{G}^{-1}\left(B_{\Gamma}^{\circ \circ}\right)$ and $B^{\circ \circ}=j_{C}^{-1}\left(B_{\Gamma}^{\circ \circ}\right)$, so that the restrictions of both $j_{G}$ and $j_{C}$ are unramified. Under an appropriate choice of the base points, the image $\mathfrak{m}_{\Gamma} \circ\left(j_{C}\right)_{*}\left(\pi_{1}\left(B^{\circ \circ}\right)\right)=\mathfrak{I m}_{C}(\Gamma) \subset \Gamma$, see Remark 3.3.4, is a subgroup of $G$. Hence $\left(j_{C}\right)_{*}\left(\pi_{1}\left(B^{\circ \circ}\right)\right) \subset\left(j_{G}\right)_{*}\left(\pi_{1}\left(B_{G}^{\circ \circ}\right)\right)=\mathfrak{m}_{\Gamma}^{-1}(G)$ and there is a lift $\tilde{\jmath}: B^{\circ \circ} \rightarrow B_{G}^{\circ \circ}$ splitting $j_{C}$. In particular, $[\Gamma: G]=\operatorname{deg} j_{G} \leqslant \operatorname{deg} j_{C}<\infty$ and the Riemann surface $B_{G}$ is well defined; compactifying all curves, one obtains a desired splitting $B_{C} \rightarrow B_{G} \rightarrow B_{\Gamma}=\mathbb{P}^{1}$.

3.5.3. Corollary. The monodromy groups $\mathfrak{I m}_{C}(\Gamma), \mathfrak{I m}_{C}(\tilde{\Gamma})$, and $\mathfrak{I m}_{C}\left(\mathbb{B}_{3}\right)$ of a non-isotrivial trigonal curve $C \subset \Sigma_{d}$ are subgroups of genus zero. In particular, they are of finite index.

Proof. It suffices to let $G=\mathfrak{I m}_{C}(\Gamma)$ in Lemma 3.5.2. The subgroup $\mathfrak{I m}_{C}\left(\mathbb{B}_{3}\right) \subset \mathbb{B}_{3}$ is also of finite index as $\mathfrak{I m}_{C}\left(\mathbb{B}_{3}\right) \cap Z\left(\mathbb{B}_{3}\right) \neq\{1\}$, see Lemma 3.3.3.

3.5.4. Remark. Note that the monodromy group $\mathfrak{I m}_{C}(\Gamma)$ of an isotrivial trigonal curve $C$ is always finite cyclic, hence of infinite index, see Subsection 4.4.

3.5.5. Definition. A curve $C_{G} \subset \Sigma_{d} \rightarrow B_{G}$ is called a universal trigonal curve corresponding to the subgroup $G \subset \Gamma$ if it has the following property: for a nonisotrivial trigonal curve $C$, one has $\mathfrak{I m}_{C}(\Gamma) \prec G$ if and only if $C$ is Nagata equivalent to a curve induced from $C_{G}$.

3.5.6. Corollary. Any genus zero subgroup $G \subset \Gamma$ admits a universal trigonal curve $C_{G} \subset \Sigma_{d} \rightarrow B_{G} \cong \mathbb{P}^{1}$ (for some $d$ depending on $G$ ) with type $\tilde{\mathbf{A}}$ singular fibers only. Such a universal curve is unique up to isomorphism.

Proof. One can take for $C_{G}$ the only trigonal curve determined by the $j$-invariant $j_{G}: B_{G} \rightarrow \mathbb{P}^{1}$ and the type specification assigning type $\mathbf{A}$ to each singular fiber. In other words, $C_{G}$ is the maximal trigonal curve determined by the skeleton $\mathrm{Sk}_{G}$, cf. Remark 3.3.5. Then, the 'if' part in Definition 3.5.5 follows from Lemmas 3.3.8 and 3.3.6, and the 'only if' part is given by Lemma 3.5.2.

3.5.7. Example. The concept of universal curve can be made very explicit in the case $G=\Gamma$. In this case $\tilde{\jmath}=j_{C}$. The 'ultimate' universal trigonal curve $C_{\Gamma} \subset \Sigma_{1}$ is the cubic in the blown-up plane $\Sigma_{1}$ given by

$$
\tilde{y}^{3}-3 \tilde{x}(\tilde{x}-1) \tilde{y}+2 \tilde{x}(\tilde{x}-1)^{2}=0,
$$

its $j$-invariant is the identity function $j_{\Gamma}(\tilde{x})=\tilde{x}$, and its singular fibers are of types $\tilde{\mathbf{A}}_{0}^{* *}$ (over $\left.\tilde{x}=0\right), \tilde{\mathbf{A}}_{1}^{*}$ (over $\tilde{x}=1$ ), and $\tilde{\mathbf{A}}_{0}^{*}$ (over $\tilde{x}=\infty$ ). It is straightforward that the Weierstraß equation (2.3.1) of any other trigonal curve $C$ is obtained from the above equation of $C_{\Gamma}$ by the substitution

$$
\tilde{x}=j_{C}(x), \quad \tilde{y}=\frac{p q}{\Delta} y,
$$

see (2.3.2), the $y$-substitution corresponding partially to a sequence of elementary Nagata transformations. 
3.6. Subgroups of $\mathbb{B}_{3}$ and $\tilde{\Gamma}$. Corollary 3.5.6 has counterparts for the braid group $\mathbb{B}_{3}$ and the extended modular group $\tilde{\Gamma}$.

3.6.1. Definition. Let $G \subset \mathbb{B}_{3}$ be a subgroup of genus zero, and let $m=\operatorname{dp} G$. A trigonal curve $C_{G} \subset \Sigma_{d} \rightarrow B_{G}$ is called a universal trigonal curve corresponding to $G$ if it has the following property: for a non-isotrivial trigonal curve $C$ one has $\mathfrak{I m}_{C}\left(\mathbb{B}_{3}\right) \prec G$ if and only if $C$ is $m$-Nagata equivalent to a curve induced from $C_{G}$.

3.6.2. Proposition. Any genus zero subgroup $G \subset \mathbb{B}_{3}$ admits a universal trigonal curve; it is unique up to isomorphism and $m$-Nagata equivalence.

Proof. It suffices to consider the universal curve $C_{G^{\prime}}$ corresponding to the projection $G^{\prime}$ of $G$ to $\Gamma$, see Corollary 3.5.6, and then change the type specification to make sure that the new $\mathbb{B}_{3}$-valued monodromy group is a subgroup of $G$.

3.6.3. Remark. Note that we do not assert that the $\mathbb{B}_{3}$-valued monodromy group $\mathfrak{I m}$ of the curve $C_{G}$ constructed in the proof coincides with $G$. The two groups have the same image in $\Gamma$, but dp $\mathfrak{I m}$ may be a proper multiple of $m$. Note, however, that one can make the two groups coincide by applying an $m$-fold Nagata transformation at a nonsingular fiber of $C_{G}$, introducing an extra singular fiber with the monodromy $\left(\sigma_{2} \sigma_{1}\right)^{3 m}$.

Given a subgroup $\tilde{G} \subset \tilde{\Gamma}$, one can consider its pull-back $G \subset \mathbb{B}_{3}$ and thus speak about a universal trigonal curve $C_{\tilde{G}}$ corresponding to $\tilde{G}$. Note that $\operatorname{dp} \tilde{G}$ equals 1 or 2 depending on whether $\tilde{G}$ does or, respectively, does not contain - id.

3.6.4. Corollary. Any genus zero subgroup $\tilde{G} \subset \tilde{\Gamma}$ admits a simple universal trigonal curve $C_{\tilde{G}}$. If - id $\notin \tilde{G}$, such a simple curve is unique up to isomorphism. If, in addition, all singular fibers of $C_{\tilde{G}}$ are of Kodaira type I, then any simple non-isotrivial trigonal curve $C$ with $\operatorname{Im}_{C}(\tilde{\Gamma}) \prec \tilde{G}$ is induced from $C_{\tilde{G}}$.

Proof. It suffices to observe that each series of 2-Nagata equivalent singular fibers contains a unique simple one, see (2.2.3), and that any curve induced from a curve with Kodaira type I singular fibers only is simple, see Lemma 2.5.2.

3.6.5. Remark. The monodromy group of the simple universal curve given by the lemma may be a proper subgroup of the pull-back of $\tilde{G}$ in $\mathbb{B}_{3}$, cf. Subsection 5.8 below.

3.7. Quotients of the fundamental group. We start with a simple lemma.

3.7.1. Lemma. Let $G \times A \rightarrow A,(g, a) \mapsto g(a)$, be a group action of a group $G$ on a group $A$, and let $K \subset A$ be a subgroup. Then the set

$$
\mathcal{I}_{K}:=\left\{g \in G \mid g(a) a^{-1} \in K \text { for any } a \in A\right\}
$$

is a subgroup of $G$. Furthermore, for each $g \in \mathcal{I}_{K}$ one has $g(K)=K$.

Proof. First, we show that $g(K) \subset K$ for any $g \in \mathcal{I}_{K}$. Indeed, if $k \in K$, then

$$
g(k)=\left(g(k) k^{-1}\right) \cdot k \in K
$$

as well. Now, if $g, h \in \mathcal{I}_{K}$ and $a \in A$, then

$$
g h(a) a^{-1}=g\left(h(a) a^{-1}\right) \cdot g(a) a^{-1} \in K,
$$

i.e., $g h \in \mathcal{I}_{K}$, and

$$
g^{-1}(a) a^{-1}=\left(g(b) b^{-1}\right)^{-1} \in K, \quad \text { where } \quad b=g^{-1}(a),
$$

i.e., $g^{-1} \in \mathcal{I}_{K}$. In particular, $g^{-1}(K) \subset K$, hence $g(K)=K$. 
3.7.2. Definition. The affine (projective) group $\pi:=\pi_{C}^{\text {afn }}$ (respectively, $\pi_{C}^{\text {proj }}$ ) of a trigonal curve $C$ is said to factor an epimorphism $\kappa: \mathfrak{F} \rightarrow G$ (or $\kappa$ is said to factor through $\pi$ ) if $\kappa$ factors through an appropriate geometric presentation of $\pi$, i.e., one has $\kappa: \mathfrak{F} \rightarrow \pi \rightarrow G$ for some geometric presentation $\mathfrak{F} \rightarrow \pi$ and some epimorphism $\pi \rightarrow G$.

Fix an epimorphism $\kappa: \mathfrak{F} \rightarrow G$ and let $K=\operatorname{Ker} \kappa \subset \mathfrak{F}$. In view of Lemma 3.7.1, this subgroup gives rise to a subgroup $\mathcal{I}_{\kappa}:=\mathcal{I}_{K} \subset \mathbb{B}_{3}$. Due to Corollary 3.4.2 (see also Remark 3.4.4), $\kappa$ factors through $\pi_{C}^{\text {afn }}$ if and only if $\operatorname{Im}_{C}\left(\mathbb{B}_{3}\right) \prec \mathcal{I}_{\kappa}$. Thus, the following two statements are immediate consequences of Corollary 3.5.3 and Proposition 3.6.2, respectively.

3.7.3. Proposition. An epimorphism $\kappa: \mathfrak{F} \rightarrow G$ factors through the affine group $\pi_{C}^{\text {afn }}$ of some non-isotrivial trigonal curve $C$ if and only if $\mathcal{I}_{\kappa} \subset \mathbb{B}_{3}$ is a subgroup of genus zero.

3.7.4. Proposition. Assume that the subgroup $\mathcal{I}_{\kappa} \subset \mathbb{B}_{3}$ corresponding to an epimorphism $\kappa: \mathfrak{F} \rightarrow G$ is of genus zero, and let $m=\operatorname{dp} \mathcal{I}_{\kappa}$. Then there exists a unique, up to isomorphism and $m$-Nagata equivalence, trigonal curve $C_{\kappa}$ with the following property: the group $\pi_{C}^{\text {afn }}$ of a non-isotrivial trigonal curve $C$ factors $\kappa$ if and only if $C$ is $m$-Nagata equivalent to a curve induced from $C_{\kappa}$.

Any curve $C_{\kappa}$ given by Proposition 3.7.4 is called a universal trigonal curve corresponding to $\kappa$. Using Corollaries 3.4.6 and 3.4.7, one arrives at the following statement.

3.7.5. Corollary. The group $\pi_{C}^{\text {afn }}$ of a non-isotrivial trigonal curve $C$ factors an epimorphism $\kappa: \mathfrak{F} \rightarrow G$ if and only if it factors the epimorphism

$$
\mathfrak{F} \rightarrow \pi_{C_{\kappa}}^{\mathrm{afn}} /\left\langle\left[\rho^{m}, \alpha\right]=1 \text { for any } \alpha \in \mathfrak{F}\right\rangle
$$

induced from a geometric presentation $\mathfrak{F} \rightarrow \pi_{C_{\kappa}}^{\text {afn }}$, where $m=\operatorname{dp} \mathcal{I}_{\kappa}$ and $C_{\kappa}$ is (any) universal curve corresponding to $\kappa$.

Due to Corollary 3.4.6, the quotient $\pi_{C_{\kappa}}^{\text {afn }} /\left[\pi_{C_{\kappa}}^{\text {afn }},\left\langle\rho^{m}\right\rangle\right]$ in Corollary 3.7.5 does not depend on the choice of a universal curve.

Finally, assume that $m=\operatorname{dp} \mathcal{I}_{\kappa}=2$, i.e., $\mathcal{I}_{\kappa}$ is the pull-back of a certain subgroup of $\tilde{\Gamma}$ not containing - id. Then Corollary 3.6.4 asserts that there is a unique, up to isomorphism, simple universal trigonal curve $C_{\kappa}$. Furthermore, combining this observation with Corollary 3.4.7, one has the following statement.

3.7.6. Corollary. Assume that $\mathrm{dp} \mathcal{I}_{\kappa}=2$ and that the simple universal curve $C_{\kappa}$ has Kodaira type I singular fibers only. Then the group $\pi_{C}^{\text {afn }}$ of a simple nonisotrivial trigonal curve $C$ factors $\kappa$ if and only if it factors a geometric presentation $\mathfrak{F} \rightarrow \pi_{C_{\kappa}}^{\text {afn }}$ of the group of $C_{\kappa}$.

\section{UNIFORM DIHEDRAL QUOTIENTS}

The principal result of this section is the proof of Theorems 1.2.2 and 1.2.3. In Subsection 4.4, we treat in details the case of isotrivial curves, which form a very spacial subclass, mostly excluded from the consideration in the previous sections. 
4.1. Quotients to be considered. Consider the epimorphism $\operatorname{deg}_{2}: \mathfrak{F} \rightarrow \mathbb{Z}_{2}$ given by $\alpha \mapsto \operatorname{deg} \alpha \bmod 2$. By the Reidemeister-Schreier algorithm (see, e.g., [12]), the kernel $K:=\operatorname{Ker}_{\operatorname{deg}_{2}}$ is freely generated by the elements $u:=\alpha_{1}^{2}, v_{1}=\alpha_{1} \alpha_{2}$, $v_{2}:=\alpha_{2} \alpha_{1}, w_{1}:=\alpha_{1} \alpha_{3}$, and $w_{2}:=\alpha_{3} \alpha_{1}$, and the conjugation $t$ by the generator of $\mathbb{Z}_{2}=\mathfrak{F} / K$ acts on the abelianization $K /[K, K]$ via $u \leftrightarrow u, v_{1} \leftrightarrow v_{2}, w_{1} \leftrightarrow w_{2}$.

The maximal generalized dihedral group through which $\operatorname{deg}_{2}$ factors is $\mathbb{D}(\mathcal{H})$, where $\mathcal{H}$ is the quotient of $K /[K, K]$ by the subgroup $\operatorname{Im}(t+\mathrm{id})$ and the square of (any) representative of the generator of $\mathbb{Z}_{2}$. (Modulo $\operatorname{Im}(t+\mathrm{id}$ ), this square does not depend on the choice of a representative.) The computation above shows that $\mathcal{H}=\mathbb{Z} a \oplus \mathbb{Z} b$, where $a:=v_{2}=-v_{1}$ and $b:=w_{1}=-w_{2}$. In other words, $\mathcal{H}$ is the abelianization of the group $\operatorname{Ker} \operatorname{deg}_{2} /\left\langle\alpha_{1}^{2}, \alpha_{2}^{2}, \alpha_{3}^{2}\right\rangle$.

It follows that any generalized dihedral quotient of $\mathfrak{F}$ that factors $\operatorname{deg}_{2}$ is of the form

$$
\mathfrak{F} \rightarrow \mathbb{D}(\mathcal{H}) \rightarrow \mathbb{D}(\mathcal{H} / H)
$$

for some subgroup $H \subset \mathcal{H}$.

4.1.2. Definition. An epimorphism $\kappa: \mathfrak{F} \rightarrow \mathbb{D}(\mathcal{H} / H), H \subset \mathcal{H}$, as in (4.1.1) is called a uniform dihedral quotient of $\mathfrak{F}$. If $\kappa$ factors through a geometric presentation $\mathfrak{F} \rightarrow \pi_{C}^{\text {afn }}$ of the fundamental group of a trigonal curve $C$, then $\mathbb{D}(\mathcal{H} / H)$ is also said to be a uniform dihedral quotient of $\pi_{C}^{\text {afn }}$, and $C$ itself is said to admit a uniform $\mathbb{D}(\mathcal{H} / H)$-covering.

We identify the group $\mathcal{H}=\mathbb{Z} a \oplus \mathbb{Z} b$ obtained in the previous paragraph with the group $\mathcal{H}$ introduced in Subsection 3.1. The kernel of the epimorphism $\mathfrak{F} \rightarrow \mathbb{D}(\mathcal{H})$ is $\mathbb{B}_{3}$-invariant; hence, the $\mathbb{B}_{3}$-action on $\mathfrak{F}$ induces a certain action on $\mathbb{D}(\mathcal{H})$.

4.1.3. Lemma. The induced $\mathbb{B}_{3}$-action on $\mathbb{D}(\mathcal{H})=\mathcal{H} \rtimes \mathbb{Z}_{2}$ splits into the product of the canonical representation of $\tilde{\Gamma}$ on $\mathcal{H}$ and the trivial action on $\mathbb{Z}_{2}$.

Proof. The induced action on $\mathcal{H}$ is well known; it can easily be found by a direct computation, $c f$. Remark 3.3.4. The splitting follows from the fact that the generator of $\mathbb{Z}_{2}$ admits an invariant representative, namely $\rho$.

4.1.4. Corollary. Any uniform dihedral quotient of the group $\pi_{C}^{\text {afn }}$ factors through the maximal uniform quotient $\pi_{C}^{\text {afn }} \rightarrow \mathbb{D}(\mathcal{Q})$, where $\mathcal{Q}=\mathcal{H} / H$ and $H$ is the sum of the images $\operatorname{Im}(g-\mathrm{id})$ over all $g \in \mathfrak{I m}_{C}(\tilde{\Gamma})$.

4.1.5. Remark. Similarly, for the standard $\mathbb{B}_{n}$-action on the free group $F_{n}$, one can consider the maximal invariant dihedral quotient $F_{n} \rightarrow \mathbb{D}(\mathcal{H}), \mathcal{H} \cong \bigoplus_{n-1} \mathbb{Z}$. Lemma 4.1.3 is in a sharp contrast with the case of $n$ even. For example, if $n=2$, the induced action on $\mathcal{H} \cong \mathbb{Z}$ is trivial, whereas the action on $\mathbb{D}(\mathcal{H})$ is not. For this reason, the maximal uniform dihedral quotient of the fundamental group of a hyperelliptic ('bigonal') curve in $\Sigma_{d}$ is not controlled by the always trivial action of the monodromy group of the curve on the kernel $\mathcal{H}$, $c f$. Subsection 3.1 below. If the monodromy group is generated by $\sigma_{1}^{s}, s>0$, the maximal dihedral quotient is easily found to be $\mathbb{D}_{2 s}$. In particular, for each integer $s>0$, there exists a reducible trigonal curve in $\Sigma_{2 s}$ whose fundamental group has a (non-uniform) quotient $\mathbb{D}_{2 s}$.

Next statement justifies our interest in uniform dihedral quotients; for more motivation, see Section 6 below. 
4.1.6. Proposition. Any generalized dihedral quotient of the fundamental group $\pi_{C}^{\text {afn }}$ of an irreducible trigonal curve $C$ is uniform.

Proof. If $C$ is irreducible, the abelianization of $\pi_{C}^{\text {afn }}$ equals $\mathbb{Z}$; hence $\pi_{C}^{\text {afn }}$ admits a unique epimorphism to $\mathbb{Z}_{2}$, and this epimorphism factors $\operatorname{deg}_{2}$. On the other hand, $\mathbb{D}(\mathcal{H})$ is the maximal generalized dihedral group through which $\operatorname{deg}_{2}$ factors.

4.2. The monodromy groups. Consider a uniform generalized dihedral quotient $\kappa: \mathfrak{F} \rightarrow \mathbb{D}(\mathcal{H} / H), H \subset \mathcal{H}$. Due to Lemma 4.1.3, the subgroup $\mathcal{I}_{\kappa} \subset \mathbb{B}_{3}$ introduced in Subsection 3.7 is the pullback of the subgroup

$$
\mathcal{I}_{H}:=\{g \in \tilde{\Gamma} \mid \operatorname{Im}(g-\mathrm{id}) \subset H\} \subset \tilde{\Gamma} .
$$

Up to the action of $\tilde{\Gamma}$, one has $H=\mathbb{Z}(m a) \oplus \mathbb{Z}(n b) \subset \mathcal{H}=\mathbb{Z} a \oplus \mathbb{Z} b$, where $m, n$ are nonnegative integers and either $m=n=0$ or $m \neq 0$ and $m \mid n$. We consider separately the following three cases.

4.2.1. The case $m=n=0$. In this case $H=0$ and $\mathcal{I}_{H}=\{1\}$. Hence, $\mathcal{I}_{\kappa} \subset \mathbb{B}_{3}$ is the central cyclic subgroup generated by $\left(\sigma_{2} \sigma_{1}\right)^{6}$.

4.2.2. The case $m \neq 0, n=0$. In this case, $\mathcal{I}_{H}$ is the infinite cyclic subgroup generated by (the image of) $\sigma_{1}^{m}$. Note that both $\left[\tilde{\Gamma}: \mathcal{I}_{H}\right]$ and $\left[\mathbb{B}_{3}: \mathcal{I}_{\kappa}\right]$ are infinite.

4.2.3. The case $m, n \neq 0, m \mid n$. In this case, $\mathcal{I}_{H}$ is the subgroup

$$
\tilde{\Gamma}_{m}(n):=\left\{\left[\begin{array}{ll}
a & b \\
c & d
\end{array}\right] \in \tilde{\Gamma} \mid\left[\begin{array}{ll}
a & b \\
c & d
\end{array}\right]=\left[\begin{array}{ll}
1 & * \\
0 & 1
\end{array}\right] \bmod n, b=0 \bmod m\right\} .
$$

(We use the notation of [3].) The image of $\tilde{\Gamma}_{m}(n)$ in $\Gamma$ is $\Gamma_{m}(n):=\Gamma_{1}(n) \cap \Gamma(m)$; note that $\Gamma_{1}(n)$ is consistent with the conventional notation. Note also that $\Gamma_{n}(n)$ is the principal congruence subgroup $\Gamma(n)$ and $\Gamma_{m}(n) \supset \Gamma(n)$ for any $m \mid n$. In particular, all groups $\mathcal{I}_{H}$ obtained in this case are congruence subgroups.

In the sequel, we use repeatedly the following obvious observation: if $g \in \tilde{\Gamma}$ and $\operatorname{det}(g-\mathrm{id})=d \neq 0$, then $\operatorname{Im}(g-\mathrm{id}) \subset \mathcal{H}$ is a subgroup of index $d$.

4.2.4. Lemma. Unless $m=1$ and $n \leqslant 3$, the group $\Gamma_{m}(n)$ is torsion free.

Proof. Any torsion element of $\Gamma$ is conjugate to either $\mathbb{X}^{ \pm 1}$ or $\mathbb{Y}$, and for any lift $g \in \tilde{\Gamma}$ of such an element the determinant $\operatorname{det}(g-$ id) takes value 1,2 , or 3 .

4.2.5. Lemma. The group $\tilde{\Gamma}_{m}(n)$ contains - id if and only if $n \leqslant 2$.

Proof. One has $\operatorname{Im}[-\mathrm{id}-\mathrm{id}]=2 \mathcal{H}$.

Recall that parabolic elements of $\tilde{\Gamma}$ are those of the form $\pm g$, where $g \in \tilde{\Gamma}$ is a unipotent matrix, i.e., $(g-\mathrm{id})^{2}=0$. Any unipotent element is conjugate to a power of $\mathbb{X} \mathbb{Y}$ (the image of $\sigma_{1}$ ). Up to $\pm \mathrm{id}$, any torsion free genus zero subgroup is generated by parabolic elements.

4.2.6. Lemma. If $n>2$ and $(m, n) \neq(1,4)$, then any parabolic element of $\tilde{\Gamma}_{m}(n)$ is unipotent.

Proof. If $g \in \tilde{\Gamma}$ is unipotent, then $\operatorname{det}(-g-\mathrm{id})=4$. 
4.2.7. Lemma. The group $\tilde{\Gamma}_{1}(4)$ has three conjugacy classes of indivisible parabolic elements, those of the images of $\sigma_{1}, \sigma_{2}^{4}$, and $\sigma_{1} \sigma_{2}^{4}$. The first two are unipotent, the last one is not.

Proof. The proof is a direct computation, see, e.g., Figure 2(c) below, where the skeleton $\Gamma / \Gamma_{1}(4)$ is shown.

4.3. Proof of Theorem 1.2.3. Consider the epimorphism $\mathbb{B}_{3} \rightarrow \mathbb{S}_{3}=\tilde{\Gamma} / \tilde{\Gamma}(2)$, and let $\mathfrak{I m}_{C}\left(\mathbb{S}_{3}\right) \subset \mathbb{S}_{3}$ be the image of $\mathfrak{I m}_{C}\left(\mathbb{B}_{3}\right)$. Geometrically, $\mathfrak{I m}_{C}\left(\mathbb{S}_{3}\right)$ is the monodromy group of the ramified covering $C \rightarrow \mathbb{P}^{1}$. Hence, $C$ is reducible if and only if $\mathfrak{I m}_{C}\left(\mathbb{S}_{3}\right)$ is not transitive, i.e., $\mathfrak{I m}_{C}(\tilde{\Gamma}) \prec \tilde{\Gamma}_{1}(2)$, and $C$ splits into three components if and only if $\mathfrak{I m}_{C}\left(\mathbb{S}_{3}\right)=\{1\}$, i.e., $\mathfrak{I m}_{C}(\tilde{\Gamma}) \subset \tilde{\Gamma}(2)$. By 4.2 .3 above, these conditions on $\mathfrak{I m}_{C}(\tilde{\Gamma})$ are equivalent to the existence of uniform $\mathbb{D}\left(\mathbb{Z}_{2}\right)$ - and $\mathbb{D}\left(\mathbb{Z}_{2} \oplus \mathbb{Z}_{2}\right)$-coverings, respectively.

According to Proposition 4.1.6, any dihedral covering of an irreducible trigonal curve is uniform. Hence, any curve admitting any $\mathbb{D}\left(\mathbb{Z}_{2}\right)$-covering is reducible.

The group $\mathbb{D}\left(\mathbb{Z}_{2} \oplus \mathbb{Z}_{2}\right) \cong \mathbb{Z}_{2} \oplus \mathbb{Z}_{2} \oplus \mathbb{Z}_{2}$ is abelian with three generators. Hence, if $\pi_{C}^{\text {afn }}$ factors to $\mathbb{D}\left(\mathbb{Z}_{2} \oplus \mathbb{Z}_{2}\right)$, its abelianization has at least three generators; from the Poincaré-Lefschetz duality (applied to the union $C \cup E \cup F \subset \Sigma_{d}$ ) it follows that $C$ must have at least three components.

4.3.1. Remark. In the proof of Lemma 2.6.5, we considered a trigonal curve $C_{x} \subset \Sigma_{1}$ with the set of singular fibers $\tilde{\mathbf{A}}_{1}^{*} \oplus 3 \tilde{\mathbf{A}}_{0}^{*}$. Any such curve can be obtained by a perturbation from a curve $C_{x}^{\prime} \subset \Sigma_{1}$ with the singular fibers $\tilde{\mathbf{A}}_{1}^{*} \oplus \tilde{\mathbf{A}}_{0}^{* *} \oplus \tilde{\mathbf{A}}_{0}^{*}$ (a nodal plane cubic projected from the point of intersection of the tangents to one of the branches at the node and one of the inflection points). The skeleton of $C_{x}^{\prime}$ is the graph $\circ \longrightarrow$ corresponding to the full modular group $\Gamma$. Hence, the $\Gamma$-valued monodromy groups of both curves are $\Gamma$ and, combining with the epimorphism $\Gamma \rightarrow \mathbb{S}_{3}$ above, one concludes that the $\mathbb{S}_{3}$-valued groups are $\mathbb{S}_{3}$, as stated.

4.4. Isotrivial curves. Isotrivial trigonal curves are easily classified and their monodromy groups are easily computed. Depending on the constant $j_{C}$, one can distinguish the following three cases.

4.4.1. The case $j_{C} \equiv 0$. One has $p(x) \equiv 0$ in (2.3.1) and (2.3.2) and the Weierstraß equation takes the form $y^{3}+2 q(x)=0$. The monodromy group $\mathfrak{I m}_{C}\left(\mathbb{B}_{3}\right)$ is the cyclic group generated by $\left(\sigma_{2} \sigma_{1}\right)^{r}$, where $r$ is the greatest common divisor of the multiplicities of the roots of $q(x)$. Hence, $\mathfrak{I m}_{C}(\tilde{\Gamma})$ is generated by $(-\mathbb{X})^{r}$, and the maximal uniform dihedral quotient that $\pi_{C}^{\text {afn }}$ may have is $\mathbb{D}\left(\mathcal{H} / \operatorname{Im}\left[(-\mathbb{X})^{r}-\mathrm{id}\right]\right)$. Note that $\operatorname{det}\left[(-\mathbb{X})^{ \pm 1}-\mathrm{id}\right]=1$ and $\operatorname{det}\left[(-\mathbb{X})^{ \pm 2}-\mathrm{id}\right]=3$, whereas $(-\mathbb{X})^{3}=-$ id. Summarizing, one has the following statements:

(1) if $r=0 \bmod 6$, the maximal uniform quotient is $\pi_{C}^{\text {afn }} \rightarrow \mathbb{D}(\mathcal{H})$;

(2) if $r=3 \bmod 6$, the maximal uniform quotient is $\pi_{C}^{\text {afn }} \rightarrow \mathbb{D}\left(\mathbb{Z}_{2} \oplus \mathbb{Z}_{2}\right)$;

(3) if $r= \pm 1 \bmod 6$, then $\pi_{C}^{\text {afn }}$ admits no nontrivial dihedral quotients;

(4) if $r= \pm 2 \bmod 6$, the maximal generalized dihedral quotient of $\pi_{C}^{\text {afn }}$ is $\mathbb{D}_{6}$.

In a sense, the curves as in (1) and (2) are degenerate and can be regarded as a special case of 4.4 .3 below. In cases (3) and (4), the curves are irreducible and all their generalized dihedral coverings are uniform.

Observe that, in case (4), adding an extra relation $\left(\sigma_{2} \sigma_{1}\right)^{6}=\mathrm{id}$, i.e., making $\rho^{2}$ a central element, one obtains an epimorphism $\pi_{C}^{\text {afn }} \rightarrow \mathbb{B}_{3}$ (given by $\alpha_{1}, \alpha_{3} \mapsto \sigma_{1}$, $\left.\alpha_{2} \mapsto \sigma_{2}\right)$. Observe also that, in this case, all roots of $q$ have even multiplicities; 
hence, one has $q=\bar{q}^{2}$ for some polynomial $\bar{q}(x)$ and the equation of the curve has the form $y^{3}+(\sqrt{2} \bar{q})^{2}=0$, i.e., the curve is of torus type, see Subsection 2.6.

4.4.2. The case $j_{C} \equiv 1$. One has $q(x) \equiv 0$ in (2.3.1) and (2.3.2) and the Weierstraß equation takes the form $y\left(y^{2}+3 p(x)\right)=0$. The monodromy group $\mathfrak{I m}_{C}\left(\mathbb{B}_{3}\right)$ is the cyclic group generated by $\left(\sigma_{2} \sigma_{1}^{2}\right)^{r}$, where $r$ is the greatest common divisor of the multiplicities of the roots of $p(x)$. Hence, $\mathfrak{I m}_{C}(\tilde{\Gamma})$ is generated by $(-\mathbb{Y})^{r}$, and the maximal uniform dihedral quotient that $\pi_{C}^{\text {afn }}$ may have is $\mathbb{D}\left(\mathcal{H} / \operatorname{Im}\left[(-\mathbb{Y})^{r}-\mathrm{id}\right]\right)$. Note that $\operatorname{det}\left[(-\mathbb{Y})^{ \pm 1}-\mathrm{id}\right]=2$, whereas $(-\mathbb{Y})^{2}=-\mathrm{id}$. Thus, one has:

(1) if $r=0 \bmod 4$, the maximal uniform quotient is $\pi_{C}^{\text {afn }} \rightarrow \mathbb{D}(\mathcal{H})$;

(2) if $r=2 \bmod 4$, the maximal uniform quotient is $\pi_{C}^{\text {afn }} \rightarrow \mathbb{D}\left(\mathbb{Z}_{2} \oplus \mathbb{Z}_{2}\right)$;

(3) if $r=1 \bmod 2$, the maximal uniform quotient is $\pi_{C}^{\text {afn }} \rightarrow \mathbb{D}_{4}$.

As in 4.4.1 above, the curves as in (1) and (2) splitting into three components can be regarded as a special case of 4.4 .3 below.

4.4.3. The case $j_{C}=$ const $\neq 0,1$. In this case, one has $p^{3} / q^{2}=$ const $\neq 0$. Hence, there is a polynomial $s(x)$ such that $p=\alpha s^{2}$ and $q=\beta s^{3}, \alpha, \beta=$ const. It is straightforward that the curve is Nagata equivalent to the union of three generatrices of the form $y=$ const in $\Sigma_{0}$. (Occasionally, a curve $C$ of this form may have $j_{C}=0$ or 1, corresponding to cases 4.4.1(1), (2) and 4.4.2(1), (2), respectively, see remarks in the corresponding sections.) Each $m$-fold Nagata transformation results in a type $\tilde{\mathbf{J}}_{m, 0}$ singular fiber. Hence, the set of singular fibers of $C$ is of the form $\bigoplus \tilde{\mathbf{J}}_{m_{i}, 0}$, and the monodromy group $\mathfrak{I m}_{C}\left(\mathbb{B}_{3}\right)$ is the cyclic group generated by $\left(\sigma_{2} \sigma_{1}\right)^{3 r}$, where $r=$ g.c.d. $\left(m_{i}\right)$. Thus, the maximal uniform dihedral quotient of $\pi_{C}^{\text {afn }}$ is $\mathbb{D}\left(\mathcal{H} / \operatorname{Im}\left[(-\mathrm{id})^{r}-\mathrm{id}\right]\right)$. One has:

(1) if $r=0 \bmod 2$, the maximal uniform quotient is $\pi_{C}^{\text {afn }} \rightarrow \mathbb{D}(\mathcal{H})$;

(2) if $r=1 \bmod 2$, the maximal uniform quotient is $\pi_{C}^{\text {afn }} \rightarrow \mathbb{D}\left(\mathbb{Z}_{2} \oplus \mathbb{Z}_{2}\right)$.

4.4.4. Definition. A trigonal curve as in 4.4.1(1), 4.4.2(1), or 4.4.3(1) above is called trivial.

Trivial curves are those 2-Nagata equivalent to the union of three 'horizontal' generatrices in $\Sigma_{0}=\mathbb{P}^{1} \times \mathbb{P}^{1}$. All such curves split into three components, their $\tilde{\Gamma}$ valued monodromy groups are trivial, and their fundamental groups admit infinite uniform dihedral quotients $\pi_{C}^{\text {afn }} \rightarrow \mathbb{D}(\mathbb{Z} \oplus \mathbb{Z})$. A trivial curve $C \subset \Sigma_{d}$ can also be characterized as follows: $d$ is even and the relatively minimal model of the double covering of $\Sigma_{d}$ ramified at $C \cup E$ is a trivial elliptic surface (elliptic curve) $\times \mathbb{P}^{1}$. The latter can also be described as the only irregular elliptic surfaces (over a rational base) or the only elliptic surfaces without singular fibers.

4.4.5. Remark. It follows that the conclusion of Theorem 1.2 .2 holds for any nontrivial isotrivial curve.

4.5. Proof of Theorem 1.2 .2 and Corollary 1.2.4. The case of isotrivial curves is considered in Subsection 4.4, see Remark 4.4.5.

Assume that $C$ is non-isotrivial. Due to Proposition 3.7.3, a uniform dihedral quotient $\mathfrak{F} \rightarrow \mathbb{D}(\mathcal{H} / H)$ factors through $\pi_{C}^{\text {afn }}$ (for some non-isotrivial curve $C$ ) if and only if the subgroup $\mathcal{I}_{H} \subset \tilde{\Gamma}$ introduced in Subsection 4.2 is of genus zero. Since the cases considered in 4.2.1 and 4.2.2 give rise to subgroups of infinite index, $\mathcal{I}_{H}$ must be of the form $\tilde{\Gamma}_{m}(n)$ for some $m, n>0, m \mid n$, the resulting quotient being $\mathbb{D}_{2 n}$ if $m=1$ or $\mathbb{D}\left(\mathbb{Z}_{m} \oplus \mathbb{Z}_{n}\right)$ if $m>1$. The genera of $\Gamma_{m}(n)$ are computed in [3], and the 
subgroups of genus zero are precisely those given by Theorem 1.2.2. Alternatively, one can observe that, with the exception of $\Gamma_{1}(1)=\Gamma, \Gamma_{1}(2)$, and $\Gamma_{1}(3)$, all groups $\Gamma_{m}(n)$ are torsion free, see Lemma 4.2.4, and refer to the list of torsion free genus zero congruence subgroups found in [14].

Corollary 1.2.4 follows from Theorems 1.2.2, 1.2.3 and Proposition 4.1.6.

\section{Properties of universal Curves}

We construct the minimal universal curves corresponding to uniform dihedral coverings, discuss their geometric properties, and, for the irreducible curves, compute their fundamental groups. The results are applied to illustrate Statements 1.2.1(3) and (4) in the introduction (see, e.g., Corollaries 5.5.1 and 5.8.1) and, in particular, to prove Theorem 1.2.5. In Remark 5.8.2, we announce a few further results concerning the Alexander polynomial.

5.1. The universal curves corresponding to dihedral quotients. Consider a uniform generalized dihedral quotient $\kappa: \mathfrak{F} \rightarrow \mathbb{D}(\mathcal{H} / H)$ and let $\mathcal{I}_{H} \subset \tilde{\Gamma}$ be the subgroup introduced in Subsection 4.2. Due to Corollary 3.6.4, there is a simple universal curve $C_{H}$ corresponding to $\mathcal{I}_{H}$. If $\mathcal{I}_{H} \not \supset-\mathrm{id}$, this curve is unique up to isomorphism; otherwise, $C_{H}$ is defined up to Nagata equivalence, and one can choose all singular fibers of $C_{H}$ to be of type $\tilde{\mathbf{A}}$.

All universal curves are maximal. Their skeletons $\mathrm{Sk}_{H} \subset S^{2}$, marked by the isomorphism type of the quotient $\mathcal{H} / H$, are shown in Figures 1 (irreducible curves) and 2 (reducible curves). We omit the trivial case $H=\mathcal{H}$; the corresponding ultimate trigonal curve is discussed in Example 3.5.7. In the figures, the sphere $S^{2}$ is represented as the quotient space $D / \partial D$, where $D$ is the disk bounded by the grey dotted circle.

To complete the description of the universal curves, we need to determine the type specifications. This is done in 5.1.1-5.1.4 below.

5.1.1. The groups $\tilde{\Gamma}_{1}(2)$ and $\tilde{\Gamma}(2)$. The skeletons are shown in Figures $2(\mathrm{a})$ and (b), respectively. Both groups contain - id, see Lemma 4.2.5, hence all singular fibers can be chosen of type $\tilde{\mathbf{A}}$. Under this convention, both curves are cubics in the blown up plane $\Sigma_{1}$. The sets of singular fibers are $\tilde{\mathbf{A}}_{1}^{*} \oplus \tilde{\mathbf{A}}_{1} \oplus \tilde{\mathbf{A}}_{0}^{*}$ for $\tilde{\Gamma}_{1}(2)$ and $3 \tilde{\mathbf{A}}_{1}$ for $\tilde{\Gamma}(2)$. In view of Theorem 1.2.3, the two curves can be regarded as, respectively, the universal reducible trigonal curve and the universal trigonal curve split into three components.

5.1.2. The group $\tilde{\Gamma}_{1}(4)$, Figure 2(c). The group does not contain - id, hence the curve is unique. Due to Lemma 4.2.7, the type specification is $\tilde{\mathbf{A}}_{3} \oplus \tilde{\mathbf{D}}_{5} \oplus \tilde{\mathbf{A}}_{0}^{*}$.

5.1.3. The group $\tilde{\Gamma}_{1}(3)$, Figure $\mathbf{1}(\mathbf{a})$. The group contains two conjugacy classes of torsion elements (the monovalent vertex in the figure), which must be $\mathbb{X}^{ \pm 1}$, as $\operatorname{det}\left(-\mathbb{X}^{ \pm 1}-\mathrm{id}\right)=1$. All parabolic elements are unipotent, see Lemma 4.2.6, corresponding to Kodaira type I singular fibers. Hence, the curve belongs to $\Sigma_{2}$ and its type specification is $\tilde{\mathbf{E}}_{6} \oplus \tilde{\mathbf{A}}_{2} \oplus \tilde{\mathbf{A}}_{0}^{*}$. This curve can be given by the affine equation $y^{3}+(y+x)^{2}=0$; hence it is of torus type.

5.1.4. Other groups. All other groups are torsion free, see Lemma 4.2.4, do not contain - id, see Lemma 4.2.5, and all their parabolic elements are unipotent, see Lemma 4.2.6. Hence, all their singular fibers are of Kodaira type I. The curves belong to $\Sigma_{d}$, where $2 d$ is the number of vertices in the corresponding skeleton. 


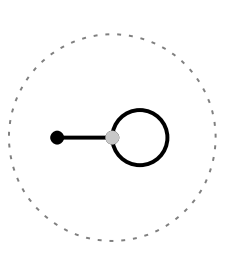

(a) $\mathbb{Z}_{3}$

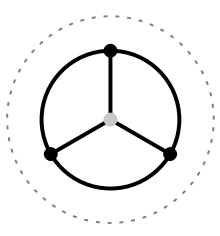

(b) $\mathbb{Z}_{3} \oplus \mathbb{Z}_{3}$

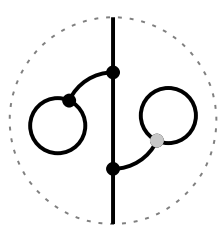

(c) $\mathbb{Z}_{5}$

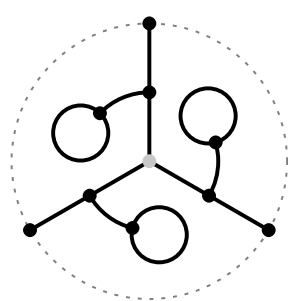

(d) $\mathbb{Z}_{7}$

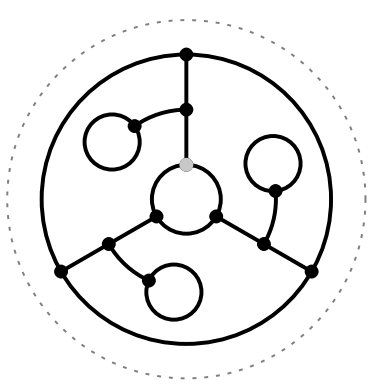

(e) $\mathbb{Z}_{9}$

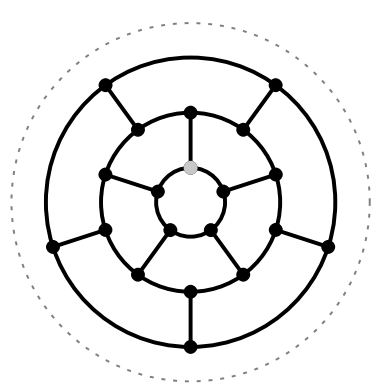

(f) $\mathbb{Z}_{5} \oplus \mathbb{Z}_{5}$

FiguRE 1. Irreducible universal curves admitting dihedral coverings

5.2. The Alexander polynomial. In this subsection, we remind the definition and basic properties of the Alexander polynomial of an algebraic curve. For more details, see A. Libgober [13].

Consider a group $G$ and a distinguished epimorphism $\kappa: G \rightarrow \mathbb{Z}$. Denote by $\mathrm{A}_{\kappa}$ the abelianization of the kernel $\operatorname{Ker} \kappa$. The conjugation by the generator of $\mathbb{Z}$ induces an automorphism $t: \mathrm{A}_{\kappa} \rightarrow \mathrm{A}_{\kappa}$, thus making $\mathrm{A}_{\kappa}$ a module over the ring $\Lambda:=\mathbb{Z}\left[t^{ \pm 1}\right]$ of Laurent polynomials in $t$. The abelian group $\mathrm{A}_{\kappa}$, regarded as a $\Lambda$-module, is called the Alexander module of $G$ (more precisely, of $\kappa$ ).

If $G$ is finitely generated, the Reidemeister-Schreier algorithm (see, e.g., [12]) asserts that $\mathrm{A}_{\kappa}$ is finitely generated over $\Lambda$. Hence, $\mathrm{A}_{\kappa} \otimes \mathbb{C}$ is finitely generated over the principal ideal domain $\Lambda_{\mathbb{C}}:=\mathbb{C}\left[t^{ \pm 1}\right]$. If $\mathrm{A}_{\kappa} \otimes \mathbb{C}$ happens to be a torsion module, its order $\Delta_{\kappa}(t) \in \Lambda_{\mathbb{C}}$ is called the Alexander polynomial of $G$. It is defined up to units, i.e., up to multiplication by $c t^{n}, c \in \mathbb{C}^{*}, n \in \mathbb{Z}$. Alternatively, under the assumptions $\mathrm{A}_{\kappa} \otimes \mathbb{C}$ is a finite dimensional vector space with an operator $t$, and $\Delta_{\kappa}$ can be defined as the characteristic polynomial of $t$. Note that, in fact, $t$ acts on the finitely generated free abelian group $\mathrm{A}_{\kappa} /$ Tors. Hence, properly normalized, $\Delta_{\kappa}$ is defined over $\mathbb{Z}$.

Any epimorphism $\rho: G^{\prime} \rightarrow G$ of groups induces an epimorphism $\mathrm{A}_{\kappa \circ \rho} \rightarrow \mathrm{A}_{\kappa}$ of their Alexander modules, which is $\Lambda$-linear. Hence one has $\Delta_{\kappa} \mid \Delta_{\kappa \circ \rho}$, provided that $\Delta_{\kappa \circ \rho}$ is defined.

Given a trigonal curve $C \subset \Sigma_{d}$, take for $\kappa: \pi_{C}^{\text {afn }} \rightarrow \mathbb{Z}$ the quotient of the degree epimorphism deg: $\mathfrak{F} \rightarrow \mathbb{Z}$. The module $\mathrm{A}_{C}:=\mathrm{A}_{\kappa}$ is called the Alexander module of $C$. Note that the central element $\rho^{d}$, see Corollary 3.4.5, is mapped to $3 d \in \mathbb{Z}$; hence, $\mathrm{A}_{C}$ is annihilated by $t^{3 d}-1$. Thus, unless $C$ is a trivial curve in $\Sigma_{0}$, the Alexander polynomial $\Delta_{C}(t):=\Delta_{\kappa}(t)$ is well defined; moreover, it is a product of cyclotomic polynomials $\Phi_{m}$ with $m \mid 3 d$. Furthermore, since $\mathrm{A}_{\mathfrak{F}} \cong \Lambda \oplus \Lambda$, each 

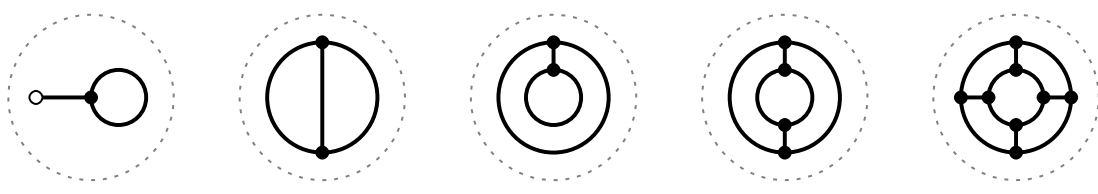

(a) $\mathbb{Z}_{2}$

(b) $\mathbb{Z}_{2} \oplus \mathbb{Z}_{2}$

(c) $\mathbb{Z}_{4}$

(d) $\mathbb{Z}_{2} \oplus \mathbb{Z}_{4}$

(e) $\mathbb{Z}_{4} \oplus \mathbb{Z}_{4}$

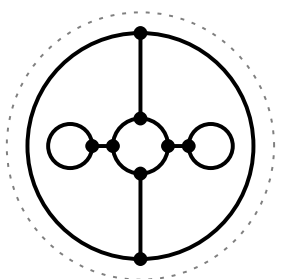

(f) $\mathbb{Z}_{8}$

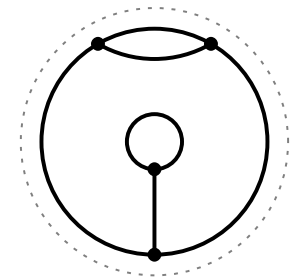

(i) $\mathbb{Z}_{6}$

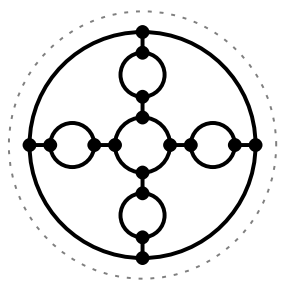

(g) $\mathbb{Z}_{2} \oplus \mathbb{Z}_{8}$

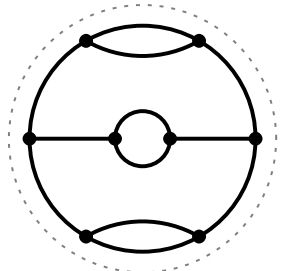

(j) $\mathbb{Z}_{2} \oplus \mathbb{Z}_{6}$

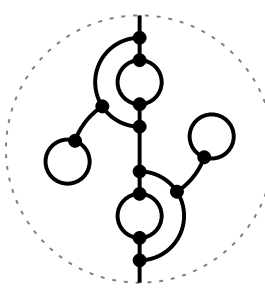

(h) $\mathbb{Z}_{10}$

FIGURE 2. Reducible universal curves admitting dihedral coverings

cyclotomic factor appears in $\Delta_{C}$ at most twice.

The following statement is essentially contained in O. Zariski [21].

5.2.1. Lemma. If a trigonal curve $C$ is irreducible, then $\Delta_{C}(t)$ is prime to $\Phi_{1}$ and to $\Phi_{m}$ for any prime power $m$.

5.2.2. Lemma. Let $m=p^{a}$ be a prime power. If $\Phi_{2 m}(t) \mid \Delta_{C}(t)$, then $C$ admits a uniform $\mathbb{D}_{2 p}$-covering. In particular, one has $p=2,3$, 5 , or 7 .

Proof. If $p=2$, the statement follows from Lemma 5.2.1, which asserts that the curve is reducible, and Theorem 1.2.3.

Assume $p$ odd. By the assumption, $\mathrm{A}^{\prime}:=\mathrm{A}_{C} / \Phi_{2 m}$ is an infinite abelian group; hence one has $\mathrm{A}^{\prime \prime}:=\operatorname{Hom}_{\mathbb{Z}}\left(\mathrm{A}^{\prime}, \mathbb{Z}_{p}\right) \neq 0$. The $\mathbb{Z}$-action on $\mathrm{A}^{\prime \prime}$ factors to an action of $\mathbb{Z}_{2 m}=\mathbb{Z}_{2} \oplus \mathbb{Z}_{m}$, the $\mathbb{Z}_{2}$ summand acting via - id. The action of the $p$-group $\mathbb{Z}_{m}$ on the $p$-group $\mathrm{A}^{\prime \prime}$ has an invariant element $\phi \neq 0$, and the semidirect product $\left(\mathrm{A}^{\prime} / \operatorname{Ker} \phi\right) \rtimes \mathbb{Z}_{2} \cong \mathbb{D}_{2 p}$ is the desired dihedral quotient.

5.2.3. Remark. At present, I do not know whether $\Phi_{14}$ can appear as a factor of the Alexander polynomial of a trigonal curve. The factors $\Phi_{6}, \Phi_{6}^{2}, \Phi_{10}$, and $\Phi_{10}^{2}$ do appear, see examples below.

5.2.4. Remark. One has $\mathcal{H}=\mathrm{A}_{\mathfrak{F}} /(t+1)$ and, in view of (4.1.1), the maximal uniform dihedral quotient of $\pi_{C}^{\text {afn }}$ is $\mathbb{D}(\mathcal{Q})$, where $\mathcal{Q}=\mathrm{A}_{C} /(t+1)$. 
5.3. Proof of Theorem 1.2.5. The implications $(2) \Longrightarrow(1)$ and $(2) \Longrightarrow(3)$ are obvious, the implication $(4) \Longrightarrow(1)$ is given by Lemma 2.6 .5 , and the implication $(3) \Longrightarrow(1)$ is given by Lemma 5.2.2. It remains to prove that $(1) \Longrightarrow(2),(4)$.

If $C$ is isotrivial, the statement is contained in Subsection 4.4, the last paragraph of 4.4.1. If $C$ is not isotrivial, it is 2-Nagata equivalent to a curve induced from the universal curve $C_{3}$ described in 5.1.3. The latter is of torus type, hence so is $C$, see Lemma 2.6.2. The braid monodromy of $C_{3}$ is found using [6] and the skeleton shown in Figure 1(a); the monodromy group is generated by $\sigma_{1}$ and $\sigma_{2}^{3}$ (the two regions in the figure) and the monodromy at infinity $\left(\sigma_{2} \sigma_{1}\right)^{6}$, see Lemma 3.3.3. The resulting affine group given by Corollary 3.4.2 is isomorphic to $\mathbb{B}_{3}$, with $\rho^{2}$ mapped to $\left(\sigma_{2} \sigma_{1}\right)^{6}$, and Corollary 3.7.5 implies that $\pi_{C}^{\text {proj }}$ factors to $\mathbb{B}_{3} /\left(\sigma_{2} \sigma_{1}\right)^{6} \cong \mathbb{Z}_{2} * \mathbb{Z}_{3}$.

5.3.1. Remark. It follows from the proof that Theorem 1.2 .5 extends to reducible curves as well, provided that in Item (1) one speaks about uniform quotients to $\mathbb{D}_{6}$ and in Item (2), about quotients $\pi_{C}^{\text {proj }} \rightarrow \mathbb{Z}_{2} * \mathbb{Z}_{3}$ that further factor to the uniform epimorphism $\pi_{C}^{\text {proj }} \rightarrow \mathbb{Z}_{2}$.

5.4. Fundamental groups of universal curves. In the rest of this section, we compute the fundamental groups of the other irreducible universal curves. Our principal goal is exploring more examples illustrating Speculation 1.2.1. Besides, this computation can also serve as a proof of the fact that the skeletons shown in Figure 1 do indeed represent the universal curves, as for each admissible pair $(m, n)$, the corresponding skeleton has the correct number of edges $\left[\Gamma: \Gamma_{m}(n)\right]$ and the resulting curve is irreducible and admits a $\mathbb{D}\left(\mathbb{Z}_{m} \oplus \mathbb{Z}_{n}\right)$-covering.

The groups obtained are analyzed using GAP [10]. To facilitate the usage of GAP, we consider the projective groups $\pi_{C}^{\text {proj }}$ rather than the affine ones $\pi_{C}^{\text {afn }}$ : these groups have finite abelianizations, hence GAP can easily find their commutants.

In each case, a presentation for $\pi_{C}^{\text {proj }}$ is given by Corollary 3.4.2, with the braid monodromy computed using the approach of [6] and the skeletons shown in Figure 1. The reference fiber $F_{0}$ is the trivalent vertex shown in the figures by a grey dot. Due to Lemma 3.3.3, in the presence of the relation at infinity $\rho^{d}=1$, one of the braid relations $\mathfrak{m}\left(\gamma_{i}\right)=$ id can be ignored. We omit the relation resulting from the outermost region of the skeleton (whenever present).

Each braid relation (resulting from an $m$-gonal region of the skeleton) has the form $\mu^{-1} \sigma_{1}^{m} \mu=$ id for some $\mu \in \mathbb{B}_{3}$. Given $\alpha, \beta \in \mathfrak{F}$ and a positive integer $m$, introduce the notation

$$
\{\alpha, \beta\}_{m}= \begin{cases}(\alpha \beta)^{k}(\beta \alpha)^{-k}, & \text { if } m=2 k \text { is even, } \\ \left((\alpha \beta)^{k} \alpha\right)\left((\beta \alpha)^{k} \beta\right)^{-1}, & \text { if } m=2 k+1 \text { is odd. }\end{cases}
$$

Then the relation $\mu^{-1} \sigma_{1}^{m} \mu=$ id is equivalent to $\mu\left\{\alpha_{1}, \alpha_{2}\right\}_{m}=1$ or, alternatively, to $\left\{\mu\left(\alpha_{1}\right), \mu\left(\alpha_{2}\right)\right\}_{m}=1$. As a special case, the relation $\sigma_{2}^{m}=\mathrm{id}$ is equivalent to $\left\{\alpha_{2}, \alpha_{3}\right\}_{m}=1$.

5.5. The group $\Gamma(3)$. The skeleton of $C$ is the tetrahedron shown in Figure 1(b); the curve is in $\Sigma_{2}$ and has four type $\tilde{\mathbf{A}}_{2}$ singular fibers. The group $\pi_{C}^{\text {proj }}$ is well known; various presentations were obtained in a number of papers. Our approach gives the relations

$$
\left\{\alpha_{1}, \alpha_{2}\right\}_{3}=\left\{\alpha_{2}, \alpha_{3}\right\}_{3}=\left\{\alpha_{3}, \alpha_{2}^{-1} \alpha_{1} \alpha_{2}\right\}_{3}=1, \quad \rho^{2}=1 .
$$

One can easily deduce that $\mathrm{A}_{C}=(\Lambda \oplus \Lambda) /\left(t^{2}-t+1\right)$; hence $\Delta_{C}=\left(t^{2}-t+1\right)^{2}$. In view of Corollary 3.7.5, one arrives at the following statement. 
5.5.1. Corollary. If a trigonal curve $C$ admits a uniform $\mathbb{D}\left(\mathbb{Z}_{3} \oplus \mathbb{Z}_{3}\right)$-covering, then $\left(t^{2}-t+1\right)^{2} \mid \Delta_{C}(t)$.

The converse of Corollary 5.5.1 is discussed in Remark 5.8.2 below.

Recall that the curve $C$ can be given by the affine equation

$$
4 y^{3}-\left(24 x^{3}+3\right) y+\left(8 x^{6}+20 x^{3}-1\right)=0 .
$$

The group $\mathbb{A}_{4} \cong \operatorname{PSL}\left(2, \mathbb{F}_{3}\right)$ of symmetries of the pair $\left(\Sigma_{2}, C\right)$ produces four nonequivalent, although isomorphic, torus structures on $C$, one for each point of the projective line $\mathbb{P}^{1}\left(\mathbb{F}_{3}\right)$ or, equivalently, for each epimorphism $\mathbb{D}\left(\mathbb{Z}_{3} \oplus \mathbb{Z}_{3}\right) \rightarrow \mathbb{D}_{6}$. In view of Lemma 2.6.2, any other curve admitting a uniform $\mathbb{D}\left(\mathbb{Z}_{3} \oplus \mathbb{Z}_{3}\right)$-covering also has at least four non-equivalent torus structures.

5.6. The groups $\Gamma_{1}(5)$ and $\Gamma_{1}(7)$. The skeletons of the universal curves are shown in Figures 1(c) and (d), respectively. The sets of singular fibers are $2 \mathbf{A}_{4} \oplus 2 \mathbf{A}_{0}^{*}$ in $\Sigma_{2}$ for $\Gamma_{1}(5)$ and $3 \mathbf{A}_{6} \oplus 3 \mathbf{A}_{0}^{*}$ in $\Sigma_{4}$ for $\Gamma_{1}(7)$.

These curves do not lead to any surprises: their affine fundamental groups are minimal possible, i.e., the semidirect products $\mathbb{Z}_{5} \rtimes \mathbb{Z}$ and $\mathbb{Z}_{7} \rtimes \mathbb{Z}$, respectively, the abelianization $\mathbb{Z}$ acting on the commutant via - id.

5.7. The group $\Gamma_{1}(9)$. The skeleton of $C$ is shown in Figure 1(e); the curve is in $\Sigma_{6}$ and its singular fibers are $3 \tilde{\mathbf{A}}_{8} \oplus 2 \tilde{\mathbf{A}}_{2} \oplus 3 \tilde{\mathbf{A}}_{0}^{*}$. The relations for $\pi_{C}^{\text {proj }}$ are

$$
\begin{gathered}
\sigma_{2}^{i}\left\{\alpha_{1}, \alpha_{2}\right\}_{9}=\sigma_{2}^{i}\left\{\alpha_{3},\left(\alpha_{2} \alpha_{1} \alpha_{2}\right)^{-1} \alpha_{1}\left(\alpha_{2} \alpha_{1} \alpha_{2}\right)\right\}_{1}=1, \quad i=0,1,2, \\
\left\{\alpha_{2}, \alpha_{3}\right\}_{3}=1, \quad \rho^{6}=1
\end{gathered}
$$

Denote this group by $G$. Using the GAP [10] commands

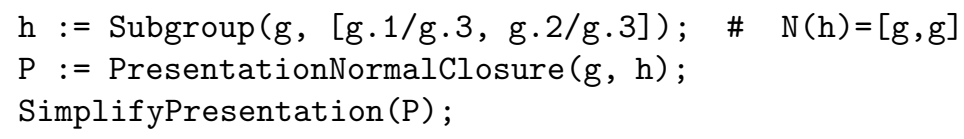

one finds that the commutant $[G, G]$ is generated by three elements $x_{2}, x_{4}, x_{6}$ that are subject to the relations (copying verbatim)

$$
x_{4}^{-1} x_{2}^{-1} x_{4} x_{2}=x_{4}^{-3} x_{2}^{3}=x_{4} x_{6}^{-1} x_{4}^{-1} x_{2} x_{6} x_{2}^{-1}=x_{2} x_{4}^{-1} x_{6} x_{4} x_{2}^{-1} x_{6}^{-1}=1,
$$

which simplify to

$$
\left(x_{2} x_{4}^{-1}\right)^{3}=\left[x_{2}, x_{4}\right]=\left[x_{6}, x_{2} x_{4}^{-1}\right]=1 .
$$

Hence, one has $[G, G]=\left\langle x_{2}, x_{6}\right\rangle \times \mathbb{Z}_{3}$, with the $\mathbb{Z}_{3}$ factor generated by $x_{2} x_{4}^{-1}$. It follows that $\mathrm{A}_{C} \cong \mathbb{Z} \oplus \mathbb{Z} \oplus \mathbb{Z}_{3}$ as a group and $\Delta_{C}(t)=t^{2}-t+1$.

A similar computation for the group $\bar{G}:=G / \rho^{2}$ shows that $[\bar{G}, \bar{G}] \cong[G, G]$; since the groups are Hopfian, the epimorphism $[G, G] \rightarrow[\bar{G}, \bar{G}]$ is an isomorphism. Due to Corollary 3.7.5, the affine fundamental group of any trigonal curve admitting a uniform $\mathbb{D}_{18}$-covering factors to $\pi_{C}^{\text {afn }} \cong\left(F_{2} \times \mathbb{Z}_{3}\right) \rtimes \mathbb{Z}$, where $F_{2}$ is the free group on two generators. 
5.8. The group $\Gamma(5)$. The skeleton is the dodecahedron shown in Figure 1(f). The curve is in $\Sigma_{10}$ and has twelve type $\tilde{\mathbf{A}}_{4}$ singular fibers. The relations for $\pi_{C}^{\text {proj }}$ are

$$
\begin{gathered}
\sigma_{2}^{i}\left\{\alpha_{1}, \alpha_{2}\right\}_{5}=\sigma_{2}^{i}\left\{\alpha_{3},\left(\alpha_{1} \alpha_{2}\right)^{-1} \alpha_{2}\left(\alpha_{1} \alpha_{2}\right)\right\}_{5}=1, \quad i=0, \ldots, 4, \\
\left\{\alpha_{2}, \alpha_{3}\right\}_{5}=1, \quad \rho^{10}=1 .
\end{gathered}
$$

Denote this group by $G$, and let $G_{\mathrm{A}}=G / \alpha_{1}^{10}$ and $\bar{G}=G / \rho^{2}$. Using GAP [10], one can easily obtain the following statements (where ' stands for the commutant):

(1) $G^{\prime} / G^{\prime \prime} \cong G_{\mathrm{A}}^{\prime} / G_{\mathrm{A}}^{\prime \prime} \cong \bigoplus_{8} \mathbb{Z}$

(2) $\bar{G}^{\prime} / \bar{G}^{\prime \prime} \cong \mathbb{Z}_{5} \oplus \mathbb{Z}_{5}$ and $\bar{G}^{\prime \prime} / \bar{G}^{\prime \prime \prime} \cong \bigoplus_{6} \mathbb{Z}_{5}$.

Since the Alexander module of $G_{\mathrm{A}}$ is obviously a quotient of both $(\Lambda \oplus \Lambda) / \Phi_{10}$ and $\mathrm{A}_{C}$, it follows from (1) that $\mathrm{A}_{C}=(\Lambda \oplus \Lambda) / \Phi_{10}$ and $\Delta_{C}(t)=\Phi_{10}^{2}(t)$. Statement (2) sheds certain light on the structure of $\bar{G}$; the group appears infinite, but I do not know a proof: my computer runs out of memory while trying to compute $\bar{G}^{\prime \prime \prime}$.

It is also immediate that the epimorphism $G^{\prime} \rightarrow \bar{G}^{\prime}$ is not an isomorphism. Hence, the monodromy group $\mathfrak{I m}_{C}\left(\mathbb{B}_{3}\right)$ of the simple universal curve is a proper subgroup of the pull-back of $\tilde{\Gamma}(5)$ in $\mathbb{B}_{3}$, cf. Remark 3.6.5.

Due to Corollary 3.7.5, the fundamental group of any trigonal curve admitting a uniform $\mathbb{D}\left(\mathbb{Z}_{5} \oplus \mathbb{Z}_{5}\right)$-covering factors to $\bar{G}$; due to Corollary 3.7.6, the group of any simple curve with this property factors to $G$.

5.8.1. Corollary. For a simple irreducible trigonal curve $C$, consider the following properties:

(1) $\pi_{C}^{\text {proj }}$ factors to $\mathbb{D}\left(\mathbb{Z}_{5} \oplus \mathbb{Z}_{5}\right)$;

(2) $\left(t^{4}-t^{3}+t^{2}-t+1\right)^{2} \mid \Delta_{C}(t)$;

(3) $\left(t^{4}-t^{3}+t^{2}-t+1\right) \mid \Delta_{C}(t)$;

(4) $\pi_{C}^{\text {proj }}$ factors to $\mathbb{D}_{10}$.

Then $(1) \Longrightarrow(2) \Longrightarrow(3) \Longrightarrow(4)$.

Proof. The first implication follows from the computation above and Corollary 3.7.6, the second one is obvious, and the last one is given by Lemma 5.2.2.

5.8.2. Remark. It can be shown that the converse implication $5.8 .1(2) \Longrightarrow(1)$, as well as the converse of Corollary 5.5.1, also hold; proof will appear elsewhere. In fact, the Alexander polynomial of a non-isotrivial trigonal curve cannot be divisible by $\Phi_{m}^{2}(t)$ unless $m=2,4,6,8$, or 10 .

5.8.3. Remark. Perturbing the type $\tilde{\mathbf{A}}_{4}$ singular fiber in the central region of Figure 1(f) to five type $\tilde{\mathbf{A}}_{0}^{*}$ fibers, one adds to the presentation above the relation $\alpha_{2}=\alpha_{3}$. Using GAP [10], it is easy to see that the Alexander polynomial of the resulting curve is $\Phi_{10}(t)$. In particular, both implications $(2) \Longrightarrow(3) \Longrightarrow(4)$ in Corollary 5.8.1 are strict.

\section{FURTher APPLICATIONS}

In Subsection 6.1, we relate the maximal uniform dihedral quotient of $\pi_{C}^{\text {afn }}$ to the homology and the torsion of the Mordell-Weil group of the covering elliptic surface. In Subsection 6.2, this relation is used in the study of another geometric property of the curve, its so called $Z$-splitting sections. Finally, in Subsections 6.3 and 6.4, we discuss the extent to which the results of the paper apply to generalized trigonal curves and prove Theorem 1.2.6. 
6.1. The topological interpretation of uniform dihedral quotients. Fix a trigonal curve $C \subset \Sigma_{d}$. Assume that $d=2 k$ is even. Then there exists a unique double covering $X \rightarrow \Sigma_{d}$ ramified at $C+E$. Denote by $\tilde{X}=\tilde{X}_{C}$ the minimal resolution of singularities of $X$, and let $\tilde{F}_{i} \subset \tilde{X}, i=0, \ldots, r$, be the preimages of the fibers $F_{i}$ introduced in Subsection 3.3, i.e., a nonsingular fiber $F_{0}$ and all singular (and possibly more nonsingular) fibers $F_{1}, \ldots, F_{r}$. The pull-backs of $C$ and $E$ in $\tilde{X}$ are identified with $C$ and $E$ themselves. Note that $\tilde{X}$ is a Jacobian elliptic surface (not necessarily relatively minimal) and $E$ is its section; one has $E^{2}=-k$ in $\tilde{X}$.

Denote $\tilde{F}_{*}=\bigcup_{i=1}^{r} \tilde{F}_{i}$ and $X^{\circ}=\tilde{X} \backslash \tilde{F}_{*}$, and let $\tilde{\gamma}_{i} \in H_{1}\left(X^{\circ} \backslash E\right)$ be the class realized by the meridian about the proper pull-back of $F_{i}, i=1, \ldots, r$; this class can also be realized by any lift of the element $\gamma_{i}$ of any geometric basis $\left\{\gamma_{1}, \ldots, \gamma_{r}\right\}$ as in Subsections 3.3 and 3.4.

Keeping in mind further applications, fix the following notation:

- $S_{C} \subset H_{2}(\tilde{X})$ is the subgroup spanned by the classes of the components of the singular fibers $\tilde{F}_{i}, i=1, \ldots, r$, and the section $E$;

- $S_{C}^{\vee}=\left(S_{C} \otimes \mathbb{Q}\right) \cap H_{2}(\tilde{X})$ is the primitive hull of $S_{C}$;

$-\mathcal{K}_{C}=S_{C}^{\vee} / S_{C}$;

- $\mathcal{Q}_{C}$ is the group $\mathcal{Q}=\mathcal{H} / H$ given by Corollary 4.1.4.

Thus, $S_{C}$ is the image of the inclusion homomorphism in $\operatorname{in}_{*}: H_{2}\left(\tilde{F}_{*} \cup E\right) \rightarrow H_{2}(\tilde{X})$, and $\mathcal{K}_{C}$ measures the imprimitivity of this image. The subgroup $S_{C}^{\vee} \subset H_{2}(\tilde{X})$ can be regarded as the 'minimal' Neron-Severi group, i.e., the Neron-Severi group of the surface $\tilde{X}$ obtained from a curve generic in its equisingular fiberwise deformation family.

6.1.1. Lemma. There are canonical isomorphisms

$$
H_{1}\left(X^{\circ}\right)=H_{1}\left(X^{\circ} \backslash E\right)=\mathcal{Q}_{C} \oplus \mathcal{Z},
$$

where $\mathcal{Z}$ is the free abelian group $\bigoplus_{i=1}^{r} \mathbb{Z} \tilde{\gamma}_{i} / \sum_{i=1}^{r} \tilde{\gamma}_{i}$. The deck translation acts via id and - id on $\mathcal{Z}$ and $\mathcal{Q}_{C}$, respectively.

Proof. The statement is immediate both algebraically, using Theorem 3.4.1, and topologically, computing the 1-homology of the locally trivial fibration $X^{\circ} \rightarrow B^{\circ}$ or $X^{\circ} \backslash E \rightarrow B^{\circ}$ using its monodromy. In both approaches, crucial is the fact that the monodromy in the homology

$$
\mathcal{H}=H_{1}(\tilde{F})=H_{1}(\tilde{F} \backslash E)=\operatorname{Ker} \operatorname{deg}_{2} /\left\langle\alpha_{1}^{2}, \alpha_{2}^{2}, \alpha_{3}^{2}\right\rangle,
$$

see Subsection 4.1, of the pull-back of a reference fiber $F$ (the homological invariant of the elliptic surface $\tilde{X}$ ) is the $\tilde{\Gamma}$-valued reduction of the braid monodromy of $C$, see Lemma 4.1.3. Note also that $\rho^{2}$ projects to $0 \in H_{1}(\tilde{F} \backslash E)$, hence the relation at infinity becomes redundant.

6.1.3. Corollary. Unless $C$ is trivial, there is an isomorphism $\mathcal{Q}_{C}=\operatorname{Ext}\left(\mathcal{K}_{C}, \mathbb{Z}\right)$.

Proof. Since $C$ is nontrivial, so is $\tilde{X}$ and $H_{1}(\tilde{X})=0$. From the Poincaré-Lefschetz duality and the exact sequence of pair $\left(\tilde{X}, \tilde{F}_{*}\right)$ one has

$$
H_{1}\left(\tilde{X}^{\circ} \backslash E\right)=\operatorname{Coker}\left[i^{*}: H^{2}(\tilde{X}) \rightarrow H^{2}\left(\tilde{F}_{*} \cup E\right)\right] .
$$

Since all homology groups involved are torsion free, the torsion of the last cokernel equals $\operatorname{Ext}\left(\mathcal{K}_{C}, \mathbb{Z}\right)$. On the other hand, due to Lemma 6.1.1 and the fact that $\mathcal{Q}_{C}$ is a torsion group, see Theorem 1.2.2, one has $\mathcal{Q}_{C}=$ Tors $H_{1}\left(\tilde{X}^{\circ} \backslash E\right)$. 
6.1.4. Remark. Since both $\mathcal{Q}_{C}$ and $\mathcal{K}_{C}$ are finite groups, the isomorphism given by Corollary 6.1.3 can be rewritten in the form $\mathcal{Q}_{C}=\operatorname{Hom}\left(\mathcal{K}_{C}, \mathbb{Q} / \mathbb{Z}\right)$ or, in other words, there is a nonsingular pairing $\mathcal{Q}_{C} \otimes \mathcal{K}_{C} \rightarrow \mathbb{Q} / \mathbb{Z}$. Hence, conversely, one has $\mathcal{K}_{C}=\operatorname{Hom}\left(\mathcal{Q}_{C}, \mathbb{Q} / Z\right)$.

6.1.5. Corollary. Assume that the trigonal curve $C \subset \Sigma_{2 k}$ is nontrivial. Then the map sending a section of $\tilde{X}$ to its homology class establishes isomorphisms Tors $M W(\tilde{X})=\mathcal{K}_{C}=\operatorname{Hom}\left(\mathcal{Q}_{C}, \mathbb{Q} / Z\right)$, where $M W$ is the Mordell-Weil group.

Proof. According to T. Shioda [17], since $H_{1}(\tilde{X})=0$, the map above establishes an isomorphism $M W(\tilde{X})=N S(\tilde{X}) / S_{C}$ and, since $N S(\tilde{X})$ is primitive in $H_{2}(\tilde{X})$, one has Tors $M W(\tilde{X})=\operatorname{Tors}\left(H_{2}(\tilde{X}) / S_{C}\right)=\mathcal{K}_{C}$. It remains to apply Corollary 6.1.3.

As a further consequence, we obtain the following known theorem.

6.1.6. Theorem (D. A. Cox, W. R. Parry [3]). The torsion of the Mordell-Weil group of a non-trivial Jacobian elliptic surface over a rational base is isomorphic to one of the groups $\mathcal{Q}$ given by Theorem 1.2.2.

6.2. $Z$-splitting sections. The notion of $Z$-splitting curves was introduced by I. Shimada in [15]; we remind the definition of [15], modifying it for the case of trigonal curves and splitting sections. Splitting, although not $Z$-splitting, sections for trigonal curves were also studied in [18] and [19].

We keep the setting and the notation of Subsection 6.1 and assume that the trigonal curve $C \subset \Sigma_{d}, d=2 k$, is nontrivial. Now, the word 'section' stands for a holomorphic section of the ruling $\Sigma_{d} \rightarrow \mathbb{P}^{1}$.

6.2.1. Definition. A section $L \subset \Sigma_{d}$ is called splitting for $C$ if the proper transform of $L$ in $\tilde{X}$ splits into two distinct components $L_{+}$and $L_{-}$. A splitting section is called pre-Z-splitting if $\left[L_{ \pm}\right] \in S_{C}^{\vee}$; in this case, the order of $\left[L_{ \pm}\right]$in the finite group $\mathcal{K}_{C}=S_{C}^{\vee} / S_{C}$ is called the class order of $L$. Finally, a pre- $Z$-splitting section is called $Z$-splitting if $\left[L_{+}\right] \neq\left[L_{-}\right]$.

Informally, $Z$-splitting sections are those that remain stable under equisingular fiberwise deformations of the curve, as one always has $S_{C}^{\vee} \subset N S(\tilde{X})$. Note though that, in the exceptional case $C \subset \Sigma_{2}$, when $\tilde{X}$ is rational, the curve may have other stable splitting sections, which are not $Z$-splitting.

Clearly, the components $L_{ \pm}$of the pull-back of a splitting section $L$ form a pair of sections of the Jacobian elliptic surface $\tilde{X}$; they are interchanged by the deck translation. Conversely, any section of $\tilde{X}$ that is not deck translation invariant projects to a splitting section for $C$. Since a section of $\tilde{X}$ is uniquely determined by its homology class, see [17], the last condition $\left[L_{+}\right] \neq\left[L_{-}\right]$in Definition 6.2.1 is redundant and any pre- $Z$-splitting section is $Z$-splitting. Finally, according to Corollary 6.1.5, a splitting section is $Z$-splitting if and only if the components $L_{ \pm}$ are torsion elements of $M W(\tilde{X})$, and the class order of $L$ equals the order of $L_{ \pm}$in $M W(\tilde{X})$. Combining, one arrives at the following statement.

6.2.2. Theorem. There is a canonical one-to-one correspondence between the set of $Z$-splitting sections of a nontrivial trigonal curve $C$ and the set of unordered pairs of distinct opposite elements of $\mathcal{K}_{C}=\operatorname{Hom}\left(\mathcal{Q}_{C}, \mathbb{Q} / \mathbb{Z}\right)$.

It follows that the number of $Z$-splitting sections for $C$ is $\frac{1}{2}\left(\left|\mathcal{Q}_{C}\right|-\left|\mathcal{Q}_{C} \otimes \mathbb{Z}_{2}\right|\right)$. In particular, an irreducible curve may have zero to four or twelve such sections. 
If $C$ is reducible, the group $\operatorname{Hom}\left(\mathcal{Q}_{C} ; \mathbb{Q} / \mathbb{Z}\right)$ has one or three elements of order 2 , see Theorem 1.2.3; they represent deck translation invariant sections of $\tilde{X}$. Clearly, these sections are the components of $C$ that are sections of $\Sigma_{d}$.

Corollary 3.6.4 gives one a very explicit description of $Z$-splitting sections: each such section $L$ is a pull-back and/or Nagata transform of a $Z$-splitting section $L^{\prime}$ for an appropriate universal curve; we will say that $L$ is induced from $L^{\prime}$. Indeed, all sections obtained in this way are $Z$-splitting, and their number equals that given by Theorem 6.2.2. Furthermore, an order $m$ element of $\mathcal{K}_{C}=\operatorname{Hom}\left(\mathcal{Q}_{C}, \mathbb{Q} / Z\right)$ can be regarded as an epimorphism $\mathcal{Q}_{C} \rightarrow \mathbb{Z}_{m}$; hence the corresponding $Z$-splitting section of class order $m$ is induced from a section for the universal curve $C_{m}$ corresponding to $\Gamma_{1}(m)$. Thus, there is a finite list of essentially distinct $Z$-splitting sections.

Any $Z$-splitting section of class order 3 is the section $y+a_{2}(x)=0$ appearing in the corresponding torus structure, see (2.6.1). For proof, it suffices to check that the section $y=0$ is $Z$-splitting for the universal curve $C_{3}=\left\{y^{3}+(y+x)^{2}=0\right\}$ corresponding to $\Gamma_{1}(3)$, see 5.1.3 and Figure 1(a).

The curve $C_{5} \subset \Sigma_{2}$, see Figure 1(c), can be given by the Weierstraß equation (2.3.1) with

$$
\begin{gathered}
-4 p(x)=x^{4}-12 x^{3}+14 x^{2}+12 x+1, \\
8 q(x)=\left(x^{2}+1\right)\left(x^{4}-18 x^{3}+74 x^{2}+18 x+1\right),
\end{gathered}
$$

and any $Z$-splitting section of class order 5 is induced from one of the two sections for $C_{5}$; they are given by $2 y=x^{2} \pm 6 x+1$. Each of these sections passes through both type $\mathbf{A}_{4}$ singular points of $C_{5}$ and is tangent to $C_{5}$ at one of these points. Note that the two $Z$-splitting sections are interchanged by the involutive symmetry $(x, y) \mapsto\left(1 / x, y / x^{2}\right)$ of $C_{5}$, constituting a single isomorphism class.

The curve $C_{4} \subset \Sigma_{2}$, see Figure 2(c), is given by $\left(y^{2}-4 x\right)(y-x-1)=0$, and its $Z$-splitting section of class order 4 is $y=2$; it passes through the type $\mathbf{A}_{3}$ point of $C_{4}$ (over $x=1$ ) and is tangent to the cusp at its type $\mathbf{D}_{5}$ point (over $x=\infty$ ).

The curve $C_{6} \subset \Sigma_{2}$, see Figure 2(i), is given by $\left(y^{2}-x\right)(y-l(x))=0$, where $16 l=-16 x^{2}+24 x+3$. Its $Z$-splitting section of class order 3 (the one arising from the torus structure) is $4 y=4 x+1$; it passes through the type $\mathbf{A}_{2}$ singular point of $C_{6}$ (over $x=\infty$ ) and is tangent to $C_{6}$ at its type $\mathbf{A}_{5}$ singular point (over $x=1 / 4$ ). The $Z$-splitting section of class order 6 is $4 y=-4 x+3$; it passes through all three singular points of $C_{6}$, including the type $\mathbf{A}_{1}$ point over $x=9 / 4$.

For the remaining class orders $7,8,9$, and 10 , I do not know explicit equations of the corresponding curves. Using Nikulin's theory of discriminant forms, it is not difficult do determine how a $Z$-splitting section should pass through the singularities of $C_{m}$; I leave this computation to the reader.

6.3. Generalized trigonal curves. By a generalized trigonal curve we mean a reduced curve $C \subset \Sigma_{d}$ with the following properties:

(1) $C$ does not contain a fiber of $\Sigma_{d}$ as a component;

(2) $C$ intersects each fiber of $\Sigma_{d}$ at three points.

Thus, unlike genuine trigonal curves defined in Subsection 2.1, a generalized trigonal curve is allowed to meet the exceptional section $E$. A singular fiber of a generalized trigonal curve $C \subset \Sigma_{d}$ is a fiber of $\Sigma_{d}$ intersecting $C+E$ geometrically at fewer than four points.

As in the case of genuine trigonal curves, see Subsection 1.2, we will consider both the projective fundamental group $\pi_{C}^{\text {proj }}:=\pi_{1}\left(\Sigma_{d} \backslash(C \cup E)\right)$ and the affine 
group $\pi_{C}^{\text {afn }}:=\pi_{1}\left(\Sigma_{d} \backslash(C \cup E \cup F)\right)$, where $F \subset \Sigma_{d}$ is a generic nonsingular fiber.

By a sequence of positive Nagata transformations, any generalized trigonal curve $C \subset \Sigma:=\Sigma_{d}$ can be transformed to a genuine trigonal curve $C^{\prime} \subset \Sigma^{\prime}:=\Sigma_{d^{\prime}}$ for some $d^{\prime} \geqslant d$. The latter is called a trigonal model of $C$; it is defined up to Nagata equivalence of trigonal curves. Let $F_{i}, i=1, \ldots, r$, be all fibers that are singular to either $C$ or $C^{\prime}$, and let $F_{0}$ be a common nonsingular fiber. (Recall that we identify fibers of the ruling and their projections to the base. Hence, we can also identify fibers of $\Sigma$ and those of $\Sigma^{\prime}$.) The Nagata transformations used establish a biholomorphism

$$
\left.\left.\Sigma \backslash\left(C \cup E \cup \bigcup_{i=0}^{r} F_{r}\right)\right) \cong \Sigma^{\prime} \backslash\left(C^{\prime} \cup E \cup \bigcup_{i=0}^{r} F_{r}\right)\right),
$$

hence an isomorphism of the respective fundamental groups. Thus, the presentation given by Theorem 3.4.1 still holds for a generalized trigonal curve $C$, assuming that the braid monodromy $\mathfrak{m}$ is defined using a trigonal model $C^{\prime}$.

6.3.1. Remark. The braid monodromy $\mathfrak{m}$ does depend on the trigonal model $C^{\prime}$. Nevertheless, the presentations given by Theorem 3.4.1 result in isomorphic groups: they differ by the lifts $\tilde{\gamma}_{i}$. A positive Nagata transformation contracting fiber $F_{i}$ multiplies $\mathfrak{m}_{i}$ by $\left(\sigma_{2} \sigma_{1}\right)^{3}$, and the new lift of $\gamma_{i}$ is $\tilde{\gamma}_{i} \rho^{-1}$.

Fix a trigonal model $C^{\prime}$ and a geometric basis $\left\{\gamma_{1}, \ldots, \gamma_{r}\right\}$ as in Subsection 3.3. Then, to each basis element $\gamma_{i}$ one can assign a slope $\varkappa_{i} \in \mathfrak{F}$, see [8]. (Note that the slope depends on both the curve $C^{\prime}$ and generator $\gamma_{i}$.) According to [8], patching back in a fiber $F_{i}$ results in an extra relation $\tilde{\gamma}_{i} \varkappa_{i}=1$. Finally, Corollary 3.4 .2 takes the following form.

6.3.2. Corollary. For a generalized trigonal curve $C$, geometric presentations of the affine and projective fundamental groups are as follows:

$$
\begin{aligned}
\pi_{C}^{\text {afn }} & =\mathfrak{F} /\left\langle\varkappa_{i} \alpha \varkappa_{i}^{-1}=\mathfrak{m}_{i}(\alpha), \quad i=1, \ldots, r, \quad \alpha \in \mathfrak{F}\right\rangle, \\
\pi_{C}^{\text {proj }} & =\pi_{C}^{\text {afn }} /\left\langle\varkappa_{r} \ldots \varkappa_{1} \rho^{d^{\prime}}\right\rangle,
\end{aligned}
$$

where the monodromies $\mathfrak{m}_{i} \in \mathbb{B}_{3}$ and slopes $\varkappa_{i} \in \mathfrak{F}, i=1, \ldots, r$, are defined using a trigonal model $C^{\prime} \subset \Sigma_{d^{\prime}}$.

It is shown in [8] that $\pi_{C}^{\text {afn }}$ is an extension of $\pi_{C}^{\text {proj }}$ by a central infinite cyclic subgroup. In particular, the commutants of the two groups are isomorphic.

6.3.3. Definition. A sequence of Nagata transformations converting a generalized trigonal curve $C$ to a trigonal model $C^{\prime}$ is called even if all slopes $\varkappa_{i}$ are of even degree. (This property is independent of the choice of a basis $\left\{\gamma_{1}, \ldots, \gamma_{r}\right\}$, see [8].) If this is the case, $C^{\prime}$ is called an even trigonal model of $C$.

It is easy to see that a trigonal model of $C$ is even if and only if it differs from the divisorial transform of $C$ by an even divisor. (For example, one can use the computation of local slopes found in [8].) A trigonal model of a genuine trigonal curve $C$ is even if and only if it is 2-Nagata equivalent to $C$; in particular, all even trigonal models of any curve $C$ are 2-Nagata equivalent. Any generalized trigonal curve admits an even trigonal model: each slope $\varkappa_{i}$ of odd degree can be corrected by a single Nagata transformation in the corresponding fiber: it multiplies $\varkappa_{i}$ by $\rho$. 
Consider the maximal uniform dihedral quotient $\mathfrak{F} \rightarrow \mathbb{D}(\mathcal{H})$, see Subsection 4.1, and, for an element $\alpha \in \mathfrak{F}$ of even degree, denote by $\tilde{\alpha} \in \mathcal{H}$ its projection to $\mathcal{H}$.

Fix a generalized trigonal curve $C$ and pick an even trigonal model $C^{\prime}$ of $C$. Denote by $\varkappa_{i}, i=1, \ldots, r$, the slopes and let $\pi_{C^{\prime}}^{\text {afn }} \rightarrow \mathbb{D}\left(\mathcal{Q}^{\prime}\right), \mathcal{Q}^{\prime}=\mathcal{H} / H$, be the maximal uniform dihedral quotient, see Corollary 4.1.4.

6.3.4. Theorem. In the notation above, the maximal uniform dihedral quotient of the affine group $\pi_{C}^{\text {afn }}$ is $\mathbb{D}\left(\mathcal{Q}^{\text {afn }}\right)$, where $\mathcal{Q}^{\text {afn }}=\mathcal{Q}^{\prime} /\left\langle 2 \tilde{\varkappa}_{i}\right\rangle, i=1, \ldots, r$. If, in addition, $C^{\prime} \subset \Sigma_{d^{\prime}}$ with $d^{\prime}=2 k$ even, then the maximal uniform dihedral quotient of the projective group $\pi_{C}^{\text {proj }}$ is $\mathbb{D}\left(\mathcal{Q}^{\text {proj }}\right)$, where $\mathcal{Q}^{\text {proj }}=\mathcal{Q}^{\text {afn }} /\left\langle\tilde{\varkappa}_{1}+\ldots+\tilde{\varkappa}_{r}\right\rangle$.

Proof. The statement follows from Corollary 6.3.2. For the projective group $\pi_{C}^{\text {proj, }}$ it suffices to consider the braid relations $\varkappa_{i} \alpha \varkappa_{i}^{-1}=\mathfrak{m}_{i}(\alpha)$ for $\alpha \in \mathfrak{F}$ an element of even degree and for $\alpha=\rho$. In the former case, one has $\tilde{\varkappa}_{i} \tilde{\alpha} \tilde{\varkappa}_{i}^{-1}=\tilde{\mathfrak{m}}_{i}(\tilde{\alpha})$, where $\tilde{\mathfrak{m}}_{i}$ is the induced automorphism of $\mathcal{H}$. Since $\mathcal{H}$ is abelian, this implies the relations $\tilde{\mathfrak{m}}_{i}=\mathrm{id}$, which define $\mathcal{Q}^{\prime}$, see Corollary 4.1.4. In the latter case, since $\mathfrak{m}_{i}(\rho)=\rho$ and $\rho^{-1} \tilde{\varkappa}_{i} \rho=-\tilde{\varkappa}_{i}$ in $\mathcal{H}$ (the definition of dihedral groups; recall that $\operatorname{deg}_{2} \rho=1$ ), one has $2 \tilde{\varkappa}_{i}=0$. For the projective group, since $d^{\prime}$ is even and $\left(\rho^{2}\right)^{\sim}=0$, the relation at infinity results in $\tilde{\varkappa}_{1}+\ldots+\tilde{\varkappa}_{r}=0$.

6.3.5. Remark. If $d^{\prime}$ is odd in Theorem 6.3.4, then the maximal uniform cyclic quotient of $\pi_{C}^{\text {proj }}$ is of odd order and $\pi_{C}^{\text {proj }}$ has no dihedral quotients.

A generalized trigonal curve is called trivial if its even trigonal models are trivial.

6.3.6. Corollary. Theorems 1.2 .2 and 1.2 .3 and Corollary 1.2 .4 hold literally for generalized trigonal curves.

6.4. Proof of Theorem 1.2.6. Blow up a singular point of $D$ of multiplicity $(\operatorname{deg} D-3)$. The result is the Hirzebruch surface $\Sigma_{1}$, and the proper transform of $D$ is a generalized trigonal curve $C \subset \Sigma_{1}$. The projection $\Sigma_{1} \rightarrow \mathbb{P}^{2}$ restricts to a biholomorphism $\Sigma_{1} \backslash(C \cup E) \cong \mathbb{P}^{2} \backslash D$, hence inducing an isomorphism $\pi_{C}^{\text {proj }}=\pi_{1}\left(\mathbb{P}^{2} \backslash D\right)$, and the statement follows from Corollary 6.3.6.

6.4.1. Example. Take for $D \subset \mathbb{P}^{2}$ the three cuspidal quartic. As is well known, $\pi_{1}\left(\mathbb{P}^{2} \backslash D\right)$ is the alternating group $\mathbb{A}_{4}$, and the maximal dihedral quotient of this group is $\mathbb{D}_{6}$. Any smooth point $P \in D$ can be taken for the distinguished 'singular' point of multiplicity $(\operatorname{deg} D-3)=1$. Hence the curve satisfies the hypotheses of Theorem 1.2.6. However, in spite of the existence of a $\mathbb{D}_{6}$-covering, Statement 1.2.5(1), none of the other statements of Theorem 1.2.5 holds for $D$.

If the point $P$ to be blown up is generic, the minimal trigonal model of $D$ is a curve $C^{\prime} \subset \Sigma_{2}$ with the set of singular fibers $3 \tilde{\mathbf{A}}_{2} \oplus \tilde{\mathbf{A}}_{1} \oplus \tilde{\mathbf{A}}_{0}^{*}$. Up to 2Nagata equivalence, $C^{\prime}$ is induced from the universal curve corresponding to $\tilde{\Gamma}_{1}(3)$, see 5.1.3. If $P$ is in the double tangent to $D$, the trigonal model is the universal curve corresponding to $\tilde{\Gamma}(3)$, see Subsection 5.5.

6.5. Speculation $\mathbf{1 . 2 . 1}$ for generalized trigonal curves. We conclude with a few remarks concerning extending Statements 1.2.1(2)-(4) to generalized trigonal curves and, in particular, to plane curves with a singular point of multiplicity $($ deg -3$)$ (which can be regarded as generalized trigonal curves in $\Sigma_{1}$ ). We keep the notation of Theorem 6.3.4: $C \subset \Sigma_{d}$ is a generalized trigonal curve, $C^{\prime} \subset \Sigma_{d^{\prime}}$ is an even trigonal model of $C$, and $\mathcal{Q}^{\prime}$ and $\mathcal{Q}^{\text {afn }}=\mathcal{Q}^{\prime} /\left\langle 2 \tilde{\varkappa}_{i}\right\rangle, i=1, \ldots, r$, are the abelian groups appearing in the maximal uniform dihedral quotients $\pi_{C^{\prime}}^{\text {afn }} \rightarrow \mathbb{D}\left(\mathcal{Q}^{\prime}\right)$ 
and $\pi_{C}^{\text {afn }} \rightarrow \mathbb{D}\left(\mathcal{Q}^{\text {afn }}\right)$. One has an epimorphism $\mathcal{Q}^{\prime} \rightarrow \mathcal{Q}^{\text {afn }} ;$ in general, it may be proper.

6.5.1. Relation to universal curves. The existence of a uniform dihedral quotient $\pi_{C}^{\text {afn }} \rightarrow \mathbb{D}\left(\mathbb{Z}_{m} \oplus \mathbb{Z}_{n}\right), m \mid n$, still implies that, up to even Nagata equivalence, $C$ is induced from the universal curve corresponding to $\tilde{\Gamma}_{m}(n)$. However, the converse is no longer true and Corollaries 3.7.5 and 3.7.6 may fail, cf. Example 6.4.1. The Alexander polynomial may also change.

6.5.2. Torus structures. Torus structures do not survive in $C$ : in general, one can only assert that the sum of $C$ with a certain linear combination of fibers is a curve of torus type (in the obvious sense), see Remark 2.6.4. For example, the three cuspidal quartic $D$ in Example 6.4.1 is not of torus type but, for any tangent $L$ to $D$, the sextic $D+2 L$ is of torus type. (After the tangency point is blown up, $L$ becomes the fiber contracted by the Nagata transformation.) If $L$ is the (only) double tangent to $D$, then $D+2 L$ admits four non-equivalent torus structures, as in this case the trigonal model of $D$ is the universal curve corresponding to $\tilde{\Gamma}(3)$, see Subsection 5.5.

6.5.3. $Z$-splitting sections. Clearly, the $Z$-splitting sections for $C$ are precisely the transforms of those for $C^{\prime}$, the correspondence preserving the class order; in fact, under an even sequence of Nagata transformations, the corresponding double coverings $\tilde{X}$ and $\tilde{X}^{\prime}$ differ at most by a few blow-ups at nonsingular points. Hence, the $Z$-splitting sections of $C$ are enumerated by pairs of distinct opposite elements of $\operatorname{Hom}\left(\mathcal{Q}^{\prime}, \mathbb{Q} / \mathbb{Z}\right)$, see Theorem 6.2 .2 , but, in general, not of $\operatorname{Hom}\left(\mathcal{Q}^{\text {afn }}, \mathbb{Q} / \mathbb{Z}\right)$. It may happen that $C$ has $Z$-splitting sections but $\mathcal{Q}^{\text {afn }}=0$, i.e., $\pi_{C}^{\text {afn }}$ does not admit a single dihedral quotient.

\section{REFERENCES}

1. V. I. Arnol'd, A. N. Varchenko, S. M. GuseĬn-Zade, Singularities of differentiable maps, vol. I. The classification of critical points, caustics and wave fronts, Nauka, Moscow, 1982 (Russian); English translation: Monographs in Mathematics, vol. 82, Birkhäuser Boston, Inc., Boston, MA, 1985.

2. E. Artin, Theory of braids, Ann. of Math. (2) 48 (1947), 101-126.

3. D. A. Cox, W. R. Parry, Torsion in elliptic curves over $k(t)$, Compositio Math. 41 (1980), no. 3, 337-354.

4. A. Degtyarev, Fundamental groups of symmetric sextics, J. Math. Kyoto Univ. 48 (2008), no. 4, 765-792.

5. A. Degtyarev, Oka's conjecture on irreducible plane sextics, J. London Math. Soc. 78, no. 2 (2008), 329-351.

6. A. Degtyarev, Zariski k-plets via dessins d'enfants, Comment. Math. Helv. 84 (2009), no. 3, 639-671.

7. A. Degtyarev, Hurwitz equivalence of braid monodromies and extremal elliptic surfaces, arXiv: 0911.0278 (to appear).

8. A. Degtyarev, The fundamental group of a generalized trigonal curve, Osaka J. Math., arXiv:0910.0100 (to appear).

9. C. Eyral, M. Oka, On the fundamental groups of the complements of plane singular sextics, J. Math. Soc. Japan 57 (2005), no. 1, 37-54.

10. The GAP Group, GAP_Groups, Algorithms, and Programming, Version 4.4.10, 2007, (http: //www.gap-system.org).

11. E. R. van Kampen, On the fundamental group of an algebraic curve, Amer. J. Math. 55 (1933), 255-260.

12. W. Magnus, A. Karrass, D. Solitar, Combinatorial group theory. Presentations of groups in terms of generators and relations, Second revised edition, Dover Publications, Inc., New York, 1976. 
13. A. Libgober, Alexander polynomial of plane algebraic curves and cyclic multiple planes, Duke Math. J. 49 (1982), no. 4, 833-851.

14. A. Sebbar, Torsion-free genus zero congruence subgroups of $\mathrm{PSL}_{2}(\mathbb{R})$, Duke Math. J. 110 (2001), no. 2, 377-396.

15. I. Shimada, Lattice Zariski k-ples of plane sextic curves and Z-splitting curves for double plane sextics, Michigan Math. J., arXiv:0903.3308 (to appear).

16. G. Shimura, Introduction to the arithmetic theory of automorphic functions, Kanô Memorial Lectures, No. 1. Publications of the Mathematical Society of Japan, No. 11, Iwanami Shoten, Publishers, Tokyo; Princeton University Press, Princeton, NJ, 1971, pp. xiv+267.

17. T. Shioda, On the Mordell-Weil lattices, Comment. Math. Univ. St. Pauli 39 (1990), no. 2, $211-240$

18. T. Shioda, H. Usui, Fundamental invariants of Weyl groups and excellent families of elliptic curves, Comment. Math. Univ. St. Pauli 41 (1992), 169-217.

19. H. Tokunaga, Splitting curves on a rational ruled surface, the Mordell-Weil groups of hyperelliptic fibrations and Zariski pairs, arXiv:0905.0047 (to appear).

20. O. Zariski, On the problem of existence of algebraic functions of two variables possessing a given branch curve, Amer. J. Math. 51 (1929), 305-328.

21. O. Zariski, On the irregularity of cyclic multiple planes, Ann. Math. 32 (1931), 485-511.

Department of Mathematics,

BiLKENT UNiversity,

06800 Ankara, Turkey

E-mail address: degt@fen.bilkent.edu.tr 